\title{
Determining the helicity structure of third generation resonances
}

\author{
Andreas Papaefstathiou ${ }^{a}$ and Kazuki Sakurai ${ }^{b}$ \\ ${ }^{a}$ Institut für Theoretische Physik, Universität Zürich, \\ Wintherturerstrasse 190, CH-8057 Zürich, Switzerland \\ ${ }^{b}$ Deutsches Elektronen Synchrotron DESY, \\ Notkestrasse 85, D-22607 Hamburg, Germany \\ E-mail: andreasp@physik.uzh.ch, kazuki.sakurai@desy.de
}

ABSTRACT: We examine methods that have been proposed for determining the helicity structure of decays of new resonances to third generation quarks and/or leptons. We present analytical and semi-analytical predictions and assess the applicability of the relevant variables in realistic reconstruction scenarios using Monte Carlo-generated events, including the effects of QCD radiation and multiple parton interactions, combinatoric ambiguities and fast detector simulation.

Keywords: Beyond Standard Model, Hadronic Colliders, Heavy Quark Physics ARXIV EPRINT: 1112.3956 


\section{Contents}

1 Introduction $\quad 1$

2 Variable definitions $\quad 3$

2.1 Daughter-to-parent energy ratios, $x_{p, i} \quad 3$

2.2 Semi-leptonic top variable, $u \quad 4$

3 Applications $\quad 7$

$\begin{array}{lll}3.1 & \text { Flavour-changing } Z^{\prime} & 7\end{array}$

$\begin{array}{lll}3.1 .1 & \text { Parton-level results } & 7\end{array}$

3.1.2 Simulation and reconstruction $\quad 8$

$\begin{array}{lll}3.2 Z^{\prime} \rightarrow \tau^{+} \tau^{-} & 13\end{array}$

$\begin{array}{lll}3.3 & \text { Third-generation leptoquark pair-production } & 16\end{array}$

$\begin{array}{lll}\text { 3.3.1 Parton-level results } & 18\end{array}$

$\begin{array}{lll}\text { 3.3.2 Simulation and reconstruction } & 20\end{array}$

4 Conclusions $\quad 24$

A Angular variables $\quad 26$

$\begin{array}{lll}\text { A.1 Definitions } & 26\end{array}$

A.2 Angular variables in the $Z^{\prime}$ flavour-changing model 26

$\begin{array}{ll}\text { B Detector-level smearing } & 26\end{array}$

C Alternative leptoquark decay: $S_{X Y} \rightarrow t_{R} \bar{\tau}_{L}, t_{L} \bar{\tau}_{R} \quad 28$

$\begin{array}{ll}\text { D Matrix element for polarised top decay } & 30\end{array}$

$\begin{array}{lll}\text { E Finite mass effects on top polarisation } & 31\end{array}$

E.1 Production polarisation 31

E.2 $\beta$ distributions from Monte Carlo 35

\section{Introduction}

The third generation is thought to be intimately connected to the mechanism responsible for electroweak symmetry breaking due to the comparatively large top, tau and bottom Yukawa couplings. New, third generation resonances are further favoured in comparison to those of the first and second generation by flavour constraints, coming from experiments investigating flavour-changing processes [1]. 
Collider searches for new resonances decaying to third generation quarks or leptons (or both in the case of leptoquarks) are particularly challenging. If the new resonance decays leptonically to either a $\tau$ or $\nu_{\tau}$, the final state necessarily possesses missing energy, which prevents us from directly reconstructing the events. If it decays to $b$ quarks, the signal has to compete with the high QCD cross sections, which can imitate the topology. In the case that the new resonance decays to top quarks, these may subsequently decay to several jets (for the hadronic decay), or to a jet plus lepton and missing energy (for the semi-leptonic decay). Both top quark decay modes require further reconstruction, where combinatorial ambiguities, QCD backgrounds and the unknown neutrino momenta can be problematic.

Despite the aforementioned difficulties, third generation resonances provide us with an interesting opportunity. The decays of the resulting third generation fermions (apart from $\nu_{\tau}$ ) may allow us to determine the chirality structure of their couplings to the new resonance. To fully determine the underlying theory and reconstruct the Lagrangian terms, determination of the coupling structure, as well as the spin of the new resonance, is necessary. The determination of the helicity of top quarks and $\tau$ leptons is made possible by the fact that the angular distributions of their daughter particles are highly correlated to the helicity of the parents. Determination of the helicity of top quarks has been investigated theoretically in detail [2-4]. Several variables have been proposed and QCD corrections to these have been calculated. Similar variables have also been proposed for $\tau$ leptons [5-7]. The $b$ quark hadronizes before decay and produces $B$ mesons which have relatively long lifetimes and thus produce displaced vertices. This allows for tagging those jets that originate from $b$ quarks but washes away the effect of its helicity from the angular distributions of the associated daughter particles. Thus, the helicity couplings of resonances containing a $b$ quark has to be inferred by first determining the spin of the parent and sister particles.

In this paper we investigate the feasibility of helicity measurement at the Large Hadron Collider (LHC) for top quarks and $\tau$ leptons resulting from the decays of new third generation resonances. We first review the variables that have been previously defined for top and $\tau$ decays and reproduce the relevant distributions, comparing them to results from a general-purpose Monte Carlo event generator, HeRwiG++ [8] (section 2). ${ }^{1}$ In the case of hadronic top quark decays, the variables have thus far mostly been considered in the highly-boosted case. We relax this approximation and attempt to determine their usefulness in more realistic reconstruction situations. For simplicity, in sections 3.1 and 3.2 respectively, we examine the applicability of these methods to a model containing a new heavy vector boson, a $Z^{\prime}$, which possesses decays to the third generation: either to a top and a light quark (specifically $t \bar{u}, \bar{t} u)^{2}$ or a pair of $\tau$ leptons. We consider reconstruction of these topologies using mass-shell constraints which lead to polynomial equations. In the latter case we also employ the $\tau$ lepton decay vertex information. Subsequently, in section 3.3, we consider the more challenging case of a third-generation leptoquark model, focusing on pair-production of these followed by decays to a top quark and a $\tau$ lepton. The reconstruction technique we employ is related to those presented in ref. [13]. Finally, we present our conclusions in section 4 .

\footnotetext{
${ }^{1}$ In appendix A we also present a set of angular variables that complement the energy fraction variables given in the literature thus far.

${ }^{2}$ Note that such models have been proposed as possible explanations to the Tevatron $t \bar{t}$ asymmetry [9-12].
} 


\section{Variable definitions}

In the decays of top quarks and $\tau$ leptons, the distribution of the cosine of the angle between the spin axis of the parent particle $k$ and the daughter particle $i, \cos \theta_{i}$, is given by:

$$
P\left(\cos \theta_{i}\right)=\frac{1}{2}\left(1+P_{k} \kappa_{i} \cos \theta_{i}\right),
$$

where $P_{k}= \pm 1$ corresponds to the spin-up or spin-down states of the parent respectively and $\kappa_{i}$ is known as the 'resolving power' of $i$. The decay product $i$ can also be taken to be a jet, i.e. containing more than one particle. The resolving power has been calculated in higher orders and for various decay products [14-17].

\subsection{Daughter-to-parent energy ratios, $x_{p, i}$}

If tops and taus are produced as a result of the decay of a heavy resonance, their own decay products may be collimated due to their large velocities. In this case, it is useful to define the energy fraction $x_{k, i}=\mathcal{E}_{k, i} / \mathcal{E}_{k}$ between the parent particle $k(=t, \tau)$ and the one of its daughter particles, $i$, rather than the angle between them. These are formed in the laboratory frame for both the tau leptons and top quarks as:

$$
\begin{aligned}
& x_{\tau, \mathrm{jet}}=\mathcal{E}_{\mathrm{jet}} / \mathcal{E}_{\tau}, \\
& x_{\mathrm{top}, \mathrm{b}}=\mathcal{E}_{b} / \mathcal{E}_{\mathrm{top}},
\end{aligned}
$$

respectively. For brevity, we will write $x_{\text {top }}$ and $x_{\tau}$ to denote the preceding variables.

Analytic predictions for these variables can be derived using eq. (2.1), by transforming from the angular variable $\cos \theta_{i}$ (defined in the centre-of-mass frame) to the energy ratio (defined in the lab frame). In the highly-boosted cases, where the boost factor, $\beta_{k} \equiv$ $\left|\vec{p}_{k}\right| / E_{k}$, is taken to be unity, their distributions are shown in figure 1 for the $\tau$ lepton decay mode $\tau \rightarrow \pi \nu_{\tau}$ (left) and hadronic top (right). The distribution of $x_{\text {top }}$ has a cut-off at the maximum value of $x_{\mathrm{top}, \max }=1-m_{W}^{2} / m_{\mathrm{top}}^{2} \sim 0.79$ and that of $x_{\tau}$ at a minimum value of $x_{\tau, \min }=m_{\pi}^{2} / m_{\tau}^{2}$, as a result of the kinematic restrictions imposed by the mass of the $W$ and the pion respectively. In the case of the $\tau$, we show only the $\tau \rightarrow \pi \nu_{\tau}$ mode, for which $m_{\pi} \simeq 0.14 \mathrm{GeV}$, which results in a small $x_{\tau, \min }$.

The exact analytic forms of the distributions are given by $[4]:^{3}$

$$
\begin{aligned}
\frac{1}{N} \frac{\mathrm{d} N}{\mathrm{~d} x_{\tau}} & =\frac{1}{\beta_{\tau}} \frac{m_{\tau}^{2}}{m_{\tau}^{2}-m_{\text {jet }}^{2}}\left(1-\frac{1}{\beta_{\tau}} \frac{P_{\tau}\left(m_{\tau}^{2}+m_{\mathrm{jet}}^{2}\right)}{m_{\tau}^{2}-m_{\mathrm{jet}}^{2}}+\frac{1}{\beta_{\tau}} \frac{2 P_{\tau} m_{\tau}^{2}}{m_{\tau}^{2}-m_{\mathrm{jet}}^{2}} x_{\tau}\right), \\
\frac{1}{N} \frac{\mathrm{d} N}{\mathrm{~d} x_{\mathrm{top}}} & =\frac{1}{\beta_{t}} \frac{m_{\mathrm{top}}^{2}}{m_{\mathrm{top}}^{2}-m_{W}^{2}}\left(1-\frac{1}{\beta_{t}} \kappa_{b} P_{t}+\kappa_{b} P_{t} \frac{1}{\beta_{t}} \frac{2 m_{\mathrm{top}}^{2}}{m_{\mathrm{top}}^{2}-m_{W}^{2}} x_{\mathrm{top}}\right),
\end{aligned}
$$

where $P_{i}= \pm 1$ (for $i=\tau, t$ ) represent right or left helicities of the $\tau$ or top and:

$$
\kappa_{b}=-\frac{m_{\text {top }}^{2}-2 m_{W}^{2}}{m_{\text {top }}^{2}+2 m_{W}^{2}} \simeq-0.4,
$$

\footnotetext{
${ }^{3}$ Note that in [4], eq. (2.4) is missing a factor of $\frac{m_{\text {top }}^{2}}{m_{\text {top }}^{2}-m_{W}^{2}}$ in the second term. The corresponding distributions, however, appear to have been constructed with the correct formulae.
} 
is [4] the resolving power of the $b$-quark at leading order, ${ }^{4} m_{\mathrm{top}}, m_{W}$ and $m_{\tau}$ are the top quark, $W$ boson and $\tau$ lepton masses respectively. The approximation $\beta_{k} \rightarrow 1$ is almost always good for $\tau$ leptons, and with the current beyond-the-standard model (BSM) third generation limits rising as the LHC experiments produce more exclusion regions (see, e.g. $[19,20]$ ), it should be even more applicable for most BSM models that include heavy particles that decay to tops. Note that in the opposite limit, of $\beta_{t} \rightarrow 0$, the above distributions tend to delta-functions, $\delta\left(x_{k}-x_{k, 0}\right)$, since the lab frame and parent top (or tau) decay frames become identical. The values of $x_{k, 0}$, determined by the masses of the decay products in the two-body decay, are $x_{\tau, 0} \simeq\left(m_{\tau}^{2}-m_{\pi}^{2}\right) /\left(2 m_{\tau}\right)$ and $x_{\text {top }, 0} \simeq$ $\left(m_{\text {top }}^{2}-m_{W}^{2}\right) /\left(2 m_{\text {top }}\right)$ for the tau and top distributions respectively, neglecting jet and bottom quark masses.

It is obvious from eqs. (2.3) and (2.4) that the effect of $\beta_{t}<1$ is to alter the predictions for the left- and right-handed distributions. The result of this effect is shown in figure 2 , where we show the $x_{\text {top }}$ right-handed helicity distributions for fixed $\beta_{t}=1.0,0.9,0.8,0.5$ and 0.1. It is obvious that for $\beta=0.9$ and $\beta=0.8$, the variation from the $\beta_{t}=1$ line is small, whereas for $\beta_{t}=0.5$ and $\beta_{t}=0.1$, the slope and position of the lines is dramatically altered. ${ }^{5}$ This is due to the fact that the slope of eq. (2.4) is inversely proportional to $\beta_{t}^{2}$. One may then safely consider the region $\beta_{t} \gtrsim 0.9$ as the 'highly-boosted' region in the case of top quark decay. This would correspond to top quarks with energies $E_{t} \gtrsim 2.5 m_{\text {top }}$. In realistic scenarios in hadron colliders, $\beta_{t}$ is not fixed, and one would need to integrate over the allowed values to obtain the full result.

The $\tau$ lepton can decay via multiple channels and each of these channels contributes to the total distribution. We do not attempt here to reproduce its analytic form, as this would require calculating the distributions of eq. (2.3) corresponding to each decay mode and integrating over the distribution of the mass of the $\tau$ jet, $m_{\text {jet }}$, with certain weight obtained by the matrix element squared. Instead we present results constructed from the Monte Carlo-simulated decays of the $\tau$ lepton using the HERWIG++ event generator. We show the resulting distributions for the left- and right-handed highly-boosted taus in figure 3. The contributions of the multiple decay modes clearly modify the behaviour compared to that of the single decay mode which appears in figure 1.

\subsection{Semi-leptonic top variable, $u$}

In the case of semi-leptonic top decays, $t \rightarrow b \ell \nu_{\ell}$, where a $b$-jet is tagged and an electron or muon is identified, we can calculate the fraction of the visible energy carried away by the lepton, $u$ :

$$
u=\frac{\mathcal{E}_{\ell}}{\mathcal{E}_{\ell}+\mathcal{E}_{b}}
$$

\footnotetext{
${ }^{4}$ The authors of ref. [18] present a study of polarisation observables at next-to-leading order with parton showers in $H^{-} t$ and $W^{-} t$ production. They find that in those cases these observables are robust against higher-order corrections.

${ }^{5}$ Note that there is also a lower limit on allowed values of $x_{\text {top }}$ due to kinematics. The limits for arbitrary top quark boosts $\beta_{t}$ are given by $\left(1-\beta_{t}\right) \leq \frac{m_{\mathrm{top}}^{2}}{m_{\mathrm{top}}^{2}-m_{W}^{2}} x_{\mathrm{top}} \leq\left(1+\beta_{t}\right)$.
} 

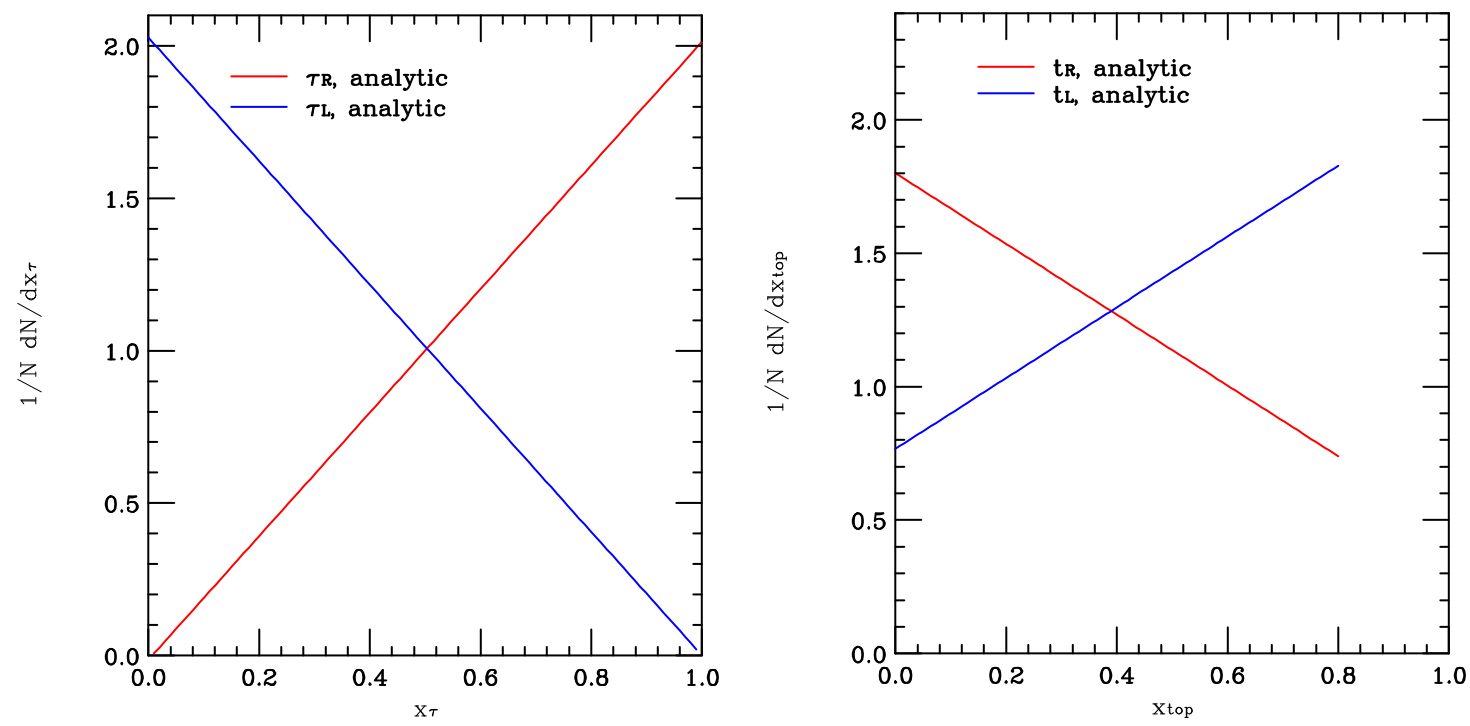

Figure 1. The predictions for the energy fractions $x_{\tau}=\mathcal{E}_{\text {jet }} / \mathcal{E}_{\tau}$ for the decay $\tau \rightarrow \pi \nu$ (left) and $x_{\text {top }}=\mathcal{E}_{b} / \mathcal{E}_{\text {top }}$ (right) in the highly-boosted cases $\left(\beta_{\tau}=1\right.$ and $\left.\beta_{t}=1\right)$.

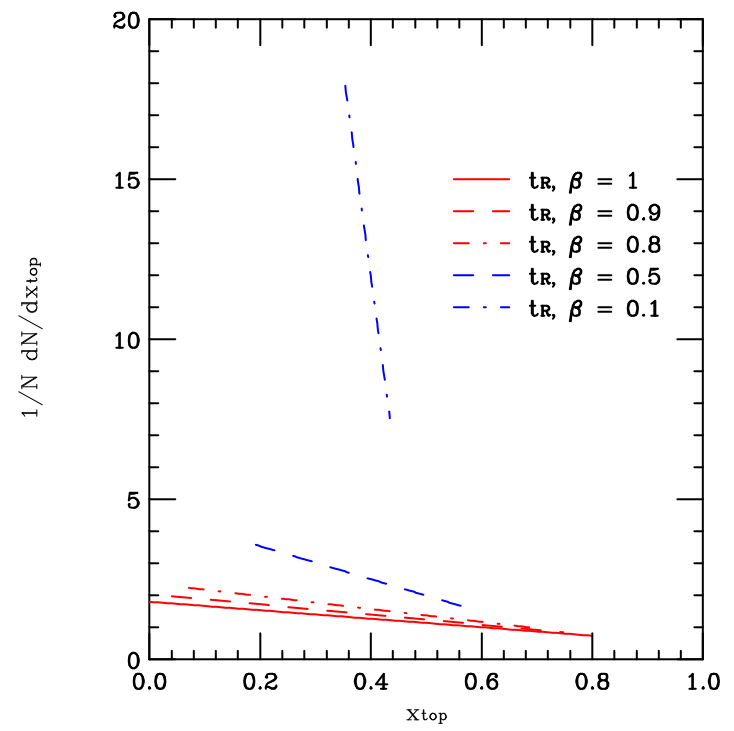

Figure 2. The predictions for the energy fraction $x_{\text {top }}=\mathcal{E}_{b} / \mathcal{E}_{\text {top }}$ for the cases $\beta_{t}=1.0,0.9,0.8,0.5$ and 0.1 .

The resulting distributions of the variable $u$ for highly-boosted $\left(\beta_{t} \rightarrow 1\right)$ [4] left- and right-handed tops are shown in figure 4. Highly-boosted Monte Carlo-generated curves are also shown for comparison, with and without final state radiation (FSR). ${ }^{6}$ The kink at $u=m_{W}^{2} / m_{\text {top }}^{2} \sim 0.215$ is due to the fact that there exists a minimum possible value of the lepton energy in the top rest frame, given by $E_{\ell, \min }=m_{W}^{2} /\left(2 m_{\mathrm{top}}\right)$, which arises when the

\footnotetext{
${ }^{6}$ Note that the curves of figure 2 in ref. [4] seem to fit the FSR on case rather than the one obtained by integrating the differential width directly, which should fit the FSR off case.
} 


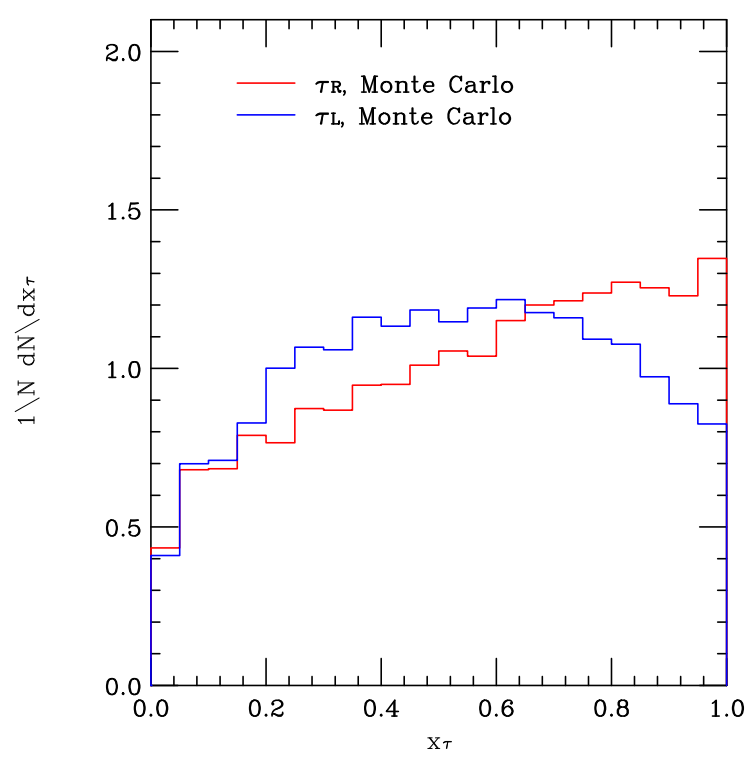

Figure 3. The Monte Carlo predictions for the energy fractions $x_{\tau}=\mathcal{E}_{\text {jet }} / \mathcal{E}_{\tau}$ for all the decay modes when the $\tau$ is highly boosted $\left(\beta_{\tau}=1\right)$.

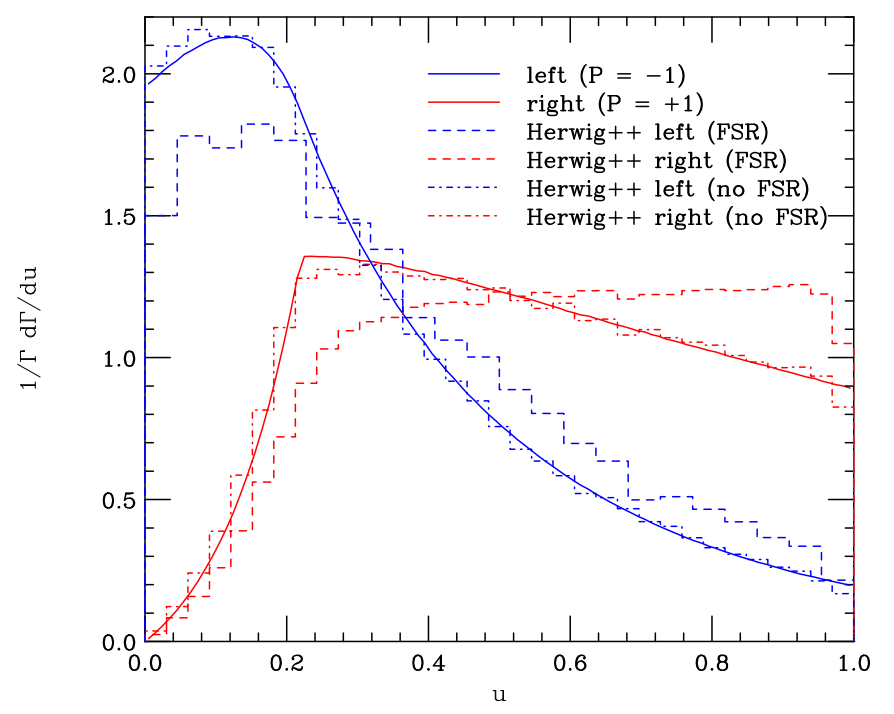

Figure 4. The distribution $1 / \Gamma \mathrm{d} \Gamma / \mathrm{d} u$ of the fraction of visible lab frame energy carried by the lepton in a highly-boosted semi-leptonic top (i.e. $\left.\beta_{t}=1\right), u=\mathcal{E}_{\ell} /\left(\mathcal{E}_{\ell}+\mathcal{E}_{b}\right)$ is shown. The blue curve and red curves represent left- and right-handed top quarks respectively.

lepton is anti-aligned with the top boost direction. The maximum value of the energy is $E_{\ell, \max }=m_{\mathrm{top}} / 2$ and arises when the lepton is aligned with the boost direction. This is clarified in figure 5, where the schematic diagram demonstrates the decay of a top in its rest frame.

It is important to note that the variable $u$ has the advantage that there is no need to explicitly reconstruct the top quarks in order to form it, even in the case of $\beta_{t} \neq 1$. As a result, it is expected to be less sensitive to the reconstruction systematics that may enter other energy fraction variables. 


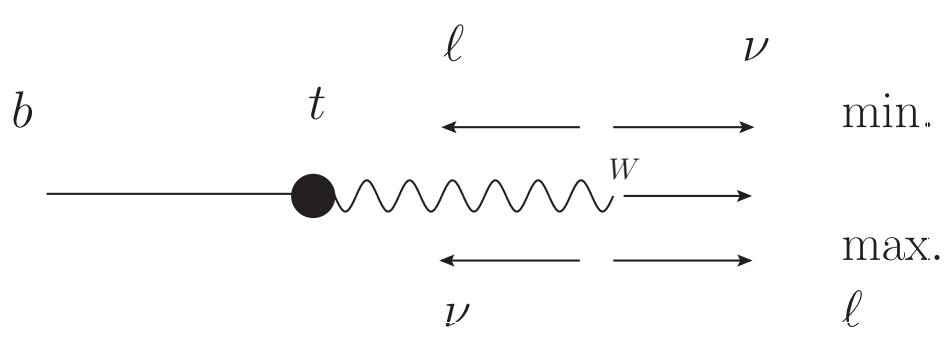

Figure 5. A schematic diagram of the decay of the top in its rest frame into a $W$ and a $b$ quark, with subsequent decay of the $W$ to a lepton and neutrino. The two configurations shown correspond to the minimum and maximum energy configurations of the lepton, corresponding to $E_{\ell, \min }=m_{W}^{2} /\left(2 m_{\mathrm{top}}\right)$ and $E_{\ell, \max }=m_{\mathrm{top}} / 2$ respectively. The minimum energy of the lepton causes the kink in the $u$ variable distribution.

\section{Applications}

We examine a model of a heavy vector boson $\left(Z^{\prime}\right)$ decaying to tops or taus and a scenario of pair-production of third generation scalar leptoquark states. In what follows we will neglect neutrino masses, and use the following values for the top quark, $W$ boson and $\tau$ lepton masses respectively: $m_{\mathrm{top}}=174.2 \mathrm{GeV}, m_{W}=80.403 \mathrm{GeV}$ and $m_{\tau}=1.777 \mathrm{GeV}$.

\subsection{Flavour-changing $Z^{\prime}$}

We first examine the application of the variables $x_{\text {top }}$ and $u$ that have been defined in the previous sections, on a model of a $Z^{\prime}$ boson that possesses flavour-changing quark couplings, described by the Lagrangian density:

$$
\begin{aligned}
\mathcal{L}= & g_{Z^{\prime}}^{t u R} Z^{\prime \mu} \bar{u}_{R} \gamma_{\mu} t_{R}+g_{Z^{\prime}}^{t u L} Z^{\prime \mu} \bar{u}_{L} \gamma_{\mu} t_{L} \\
& +g_{Z^{\prime}}^{u u R} Z^{\prime \mu} \bar{u}_{R} \gamma_{\mu} u_{R}+g_{Z^{\prime}}^{u u L} Z^{\prime \mu} \bar{u}_{L} \gamma_{\mu} u_{L}+\text { h.c. },
\end{aligned}
$$

where $g_{Z^{\prime}}^{t u L}$ and $g_{Z^{\prime}}^{t u R}$ are the flavour-changing left- and right-handed parameters that we will be varying.

It is evident that the $Z^{\prime}$ bosons of this model would be produced at a hadron collider via light $u \bar{u}$ initial states. They will decay to both light $u \bar{u}$ and $t \bar{u}$ (and $u \bar{t}$ ). After discovery of such a state, for example in dijet resonance searches, determining the helicity structure of the $u \bar{u} Z$ vertex will be challenging, if not impossible. Hence one would concentrate on determining the helicity structure of the vertex which involves the top quark.

\subsubsection{Parton-level results}

We consider a $Z^{\prime}$ described by the particular model given by the Lagrangian of eq. (3.1), of mass $1.5 \mathrm{TeV}$, choosing either a purely left-handed third generation coupling: $g_{Z^{\prime}}^{t u R}=$ $0, g_{Z^{\prime}}^{t u L}=1$ or a purely right-handed one: $g_{Z^{\prime}}^{t u R}=1, g_{Z^{\prime}}^{t u L}=0$, keeping in both cases $g_{Z^{\prime}}^{u u}=g_{Z^{\prime}}^{u u L}=1 .{ }^{7}$ We show Monte Carlo results of the distributions of the variables $x_{\text {top }}$ and $u$, for a $14 \mathrm{TeV}$ LHC, in the dashed curves in figure 6 . These results were obtained

\footnotetext{
${ }^{7}$ This mass/coupling combination is currently being marginally excluded by results presented by the LHC experiments. The aim of the present study is to assess the viability of the reconstruction variables.
} 

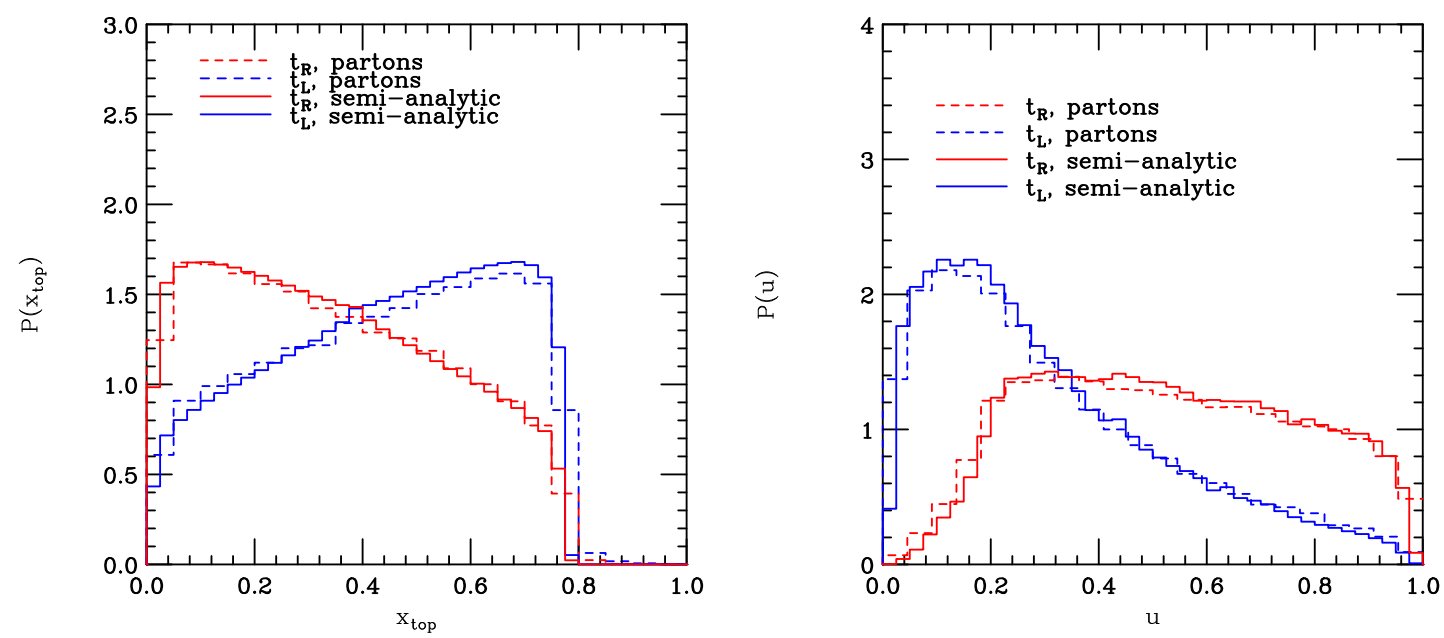

Figure 6. The $x_{\text {top }}$ (left panel) and $u$ (right panel) variables for left- or right-handed $Z^{\prime}$ bosons decaying to $u \bar{t}$ or $\bar{u}$, at a $14 \mathrm{TeV}$ LHC, obtained from parton-level events compared to a semianalytic prediction as described in the main text.

using parton-level events, ignoring initial- and final-state radiation, hadronization, and applying no rapidity or momentum cuts on the particles. The plots in figure 6 also contain semi-analytic predictions that take into account the finite boost of the top quark in the lab frame (solid curves). The semi-analytic $x_{\text {top }}$ distribution was produced by assuming that the cosine of the angle of the emitted $b$-quark in the top quark rest frame, $\cos \theta_{b}$, was distributed according to eq. (2.1) with $\kappa_{b} \simeq-0.4$, and then boosted to the lab frame according to the top quark boost $\left(\beta_{t}\right)$ distribution. The $\beta_{t}$ distribution was extracted from the Monte Carlo event generator directly, but can be fitted using a Gaussian distribution, yielding identical results (see appendix E.2). Similarly, the $u$ variable distribution was calculated first by distributing via a Monte Carlo technique the energy and $z$-momentum of the lepton in the top centre-of-mass frame ( $E_{\ell}$ and $p_{\ell}^{z}$ respectively), according to the full matrix element (see appendix D) and then boosting to the lab frame using the top quark boost distribution. The variable $u$ was then calculated by taking the ratio:

$$
u=\frac{E_{\ell}+\beta_{t} p_{\ell}^{z}}{E_{\ell}+\beta_{t} p_{\ell}^{z}+E_{b}+\beta_{t} p_{b}^{z}} .
$$

We ignore the mixing of helicities due to finite masses since the top quark is produced in association with a light quark (for which $\left.m_{Z^{\prime}} \gg m_{u}\right)^{8}$

\subsubsection{Simulation and reconstruction}

We will assume that the new resonances have been discovered, and that their mass has been measured to a satisfactory accuracy (say, $\mathcal{O}($ a few $\%)$ ). We will also assume that the spin of the new resonance has been determined by measuring the angular distributions of the

\footnotetext{
${ }^{8}$ These effects are small in the case of the $Z^{\prime}$ model but have been calculated in the leptoquark case in the following section, where the reconstruction is explained in further detail.
} 


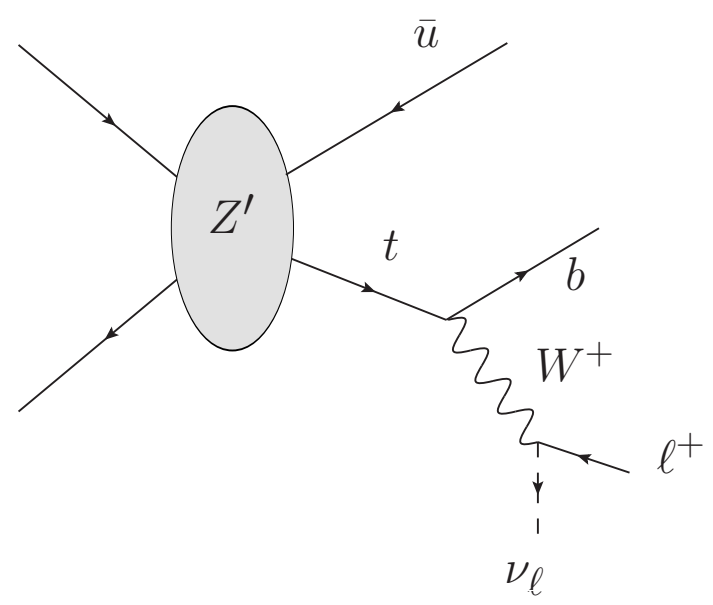

Figure 7. Production of $\bar{u} t$ from the exchange of a $Z^{\prime}$ and subsequent decay of the top into a leptonic $W$ and a $b$-jet. This mode can be fully reconstructed if one applies the $W$ mass shell condition, and chooses the solution which yields the 'best' top mass.

jets originating from the $u$ and $\bar{u}$ partons in the $u \bar{u}$ decay mode. It is necessary, however, to outline the details of the method for reconstructing events of a particular topology, for which we can form the variables we have been examining thus far at parton level.

We focus on LHC proton-proton collisions at $14 \mathrm{TeV}$, in which a $Z^{\prime}$ is exchanged, producing a $u$ (or $\bar{u}$ )-quark and an anti-top (or top), with a subsequent semi-leptonic (restricted to $e$ or $\mu$ ) decay of the top. The topology is shown in figure 7. The $u \bar{t}$ and $\bar{u} t$ decay modes account for slightly less than $\sim 50 \%$ of the total decay widths, if only one helicity (left- or right-handed) is present: $\Gamma_{Z^{\prime}, M=1.5 \mathrm{TeV}}(u \bar{t} / \bar{u} t)=236 \mathrm{GeV}$. The leading-order cross sections for the specific topology at a $14 \mathrm{TeV}$ LHC are $\sigma\left(Z^{\prime} \rightarrow t \bar{u} / \bar{t} u \rightarrow\right.$ $b \ell^{ \pm} \nu+$ jet $)=6.3 \mathrm{pb}$, again, if only one helicity is present, and including the branching ratio of the top quark to electrons or muons. We checked that the obvious irreducible background, SM QCD production of $t+q$, could be reduced to less than $\sim 1 \mathrm{pb}$, with an appropriate invariant mass cut on the visible decay products of the $t q$ pair, e.g. of around $500 \mathrm{GeV}$, with a smaller effect on the $Z^{\prime}$ signal, $\sim 60-70 \%$. We expect that other, reducible, SM backgrounds should be easier to reject in the helicity analysis.

If a $b$-jet is tagged and a high- $p_{T}$ lepton is found, along with the high- $p_{T}$ jet originating from the $u$, the information left missing to fully reconstruct the final state are the three spatial momentum components of the neutrino (assuming massless neutrino). We can obtain the transverse components of the neutrino momentum to a reasonable accuracy $(\mathcal{O}$ (few \%) ) by assuming that they are equal to the components of the missing transverse momentum. Then, the only remaining missing information is the $z$-component of the neutrino momentum. In a hadron collider we do not possess any information on the initial $z$-momentum of the system. However if we assume that the neutrino and the lepton originated from the decay of an on-shell $W$ boson, we may apply the following mass-shell condition on their four-momenta:

$$
\left(p_{\ell}+p_{\nu}\right)^{2}=m_{W}^{2}
$$


where $p_{\ell}$ and $p_{\nu}$ are the lepton and neutrino four-momenta respectively, and $m_{W}$ is the on-shell $W$ boson mass. This approximation is good, since the width of the $W$ boson is small compared to its mass $\left(\Gamma_{W} \simeq 2.14 \mathrm{GeV}\right.$ versus $\left.m_{W} \simeq 80.40 \mathrm{GeV}\right)$, and leaves us with a quadratic equation for the $z$-component of the neutrino momentum. To pick one of the two solutions, we choose the one that also yields a top mass closest to the on-shell top mass, via:

$$
m_{t, A / B}^{2}=\left(p_{\ell}+p_{\nu, A / B}+p_{b}\right)^{2},
$$

where $p_{b}$ is the $b$-jet four-momentum and $m_{t, A / B}$ is the top quark mass obtained by using the solutions for the neutrino momentum $p_{\nu, A / B}$. Once the 'best' solution is chosen, we possess all information required concerning the event, and we can thus calculate all the variables we have been examining at parton level.

The $Z^{\prime}$ model has been implemented and has been simulated using the HeRWIG++ event generator with initial- and final-state radiation turned on, as well as hadronization and the underlying event. We simulated $10 \mathrm{fb}^{-1}$ of data, a reasonable amount in a near- to mid-term LHC run at $14 \mathrm{TeV}$. The events were then processed through the Delphes detector simulation [21], where the following minimum cuts are applied to the reconstructed objects:

- $p_{T, \text { min }}$ for jets of $20.0 \mathrm{GeV}$.

- $p_{T, \text { min }}$ for electrons and muons of $10.0 \mathrm{GeV}$.

The default Delphes $b$-quark flat- $p_{T}$ tagging efficiency was replaced by a more realistic function of jet transverse momentum, $p_{T}$, which has the form

$$
P\left(p_{T, \mathrm{j}}\right)=0.08+0.006 \times p_{T, \mathrm{j}} \times \exp \left(-3 \times 10^{-5} p_{T, \mathrm{j}}^{2}\right) .
$$

See for example ref. [22] for further details. The dependence on jet pseudo-rapidity remained flat. An additional cut requiring the total missing transverse energy to be greater than $20 \mathrm{GeV}$ was applied. The rest represent the default settings present in the Delphes ATLAS detector card, where the triggering simulation has been turned off. In this analysis, and the rest of this paper, we use the anti- $k_{T}$ clustering algorithm with a radius parameter $R=0.4$ to construct jets. ${ }^{9}$

The 'best' reconstructed top mass, after detector effects, was found to possess a peak at the correct on-shell top mass, $\sim 174 \mathrm{GeV}$, with an approximately Gaussian distribution of width $\sim 20 \mathrm{GeV}$. To illustrate the effect of the Delphes detector simulation, we show a comparison of results before and after Delphes processing in figures 8 and 9 for $x_{\text {top }}$ and $u$ respectively. The comparisons between the left- and right-handed variable distributions after detector effects are shown in figure 10. It is clear that, at least with the minimum cuts, the $u$ variable performs well in discriminating between the pure left- or right-handed top quarks. The $x_{\text {top }}$ variable is less different between the two top helicities, but the difference is still statistically significant. The advantage possessed by the variable $u$ is clear: since

\footnotetext{
${ }^{9}$ It is advantageous to use a smaller radius parameter for the anti- $k_{T}$ clustering algorithm than the Delphes default one of $R=0.7$, since underlying event and pile-up contaminations are expected to be approximately proportional to $R^{2}$. See ref. [23] for further details on jet algorithms and the underlying event.
} 

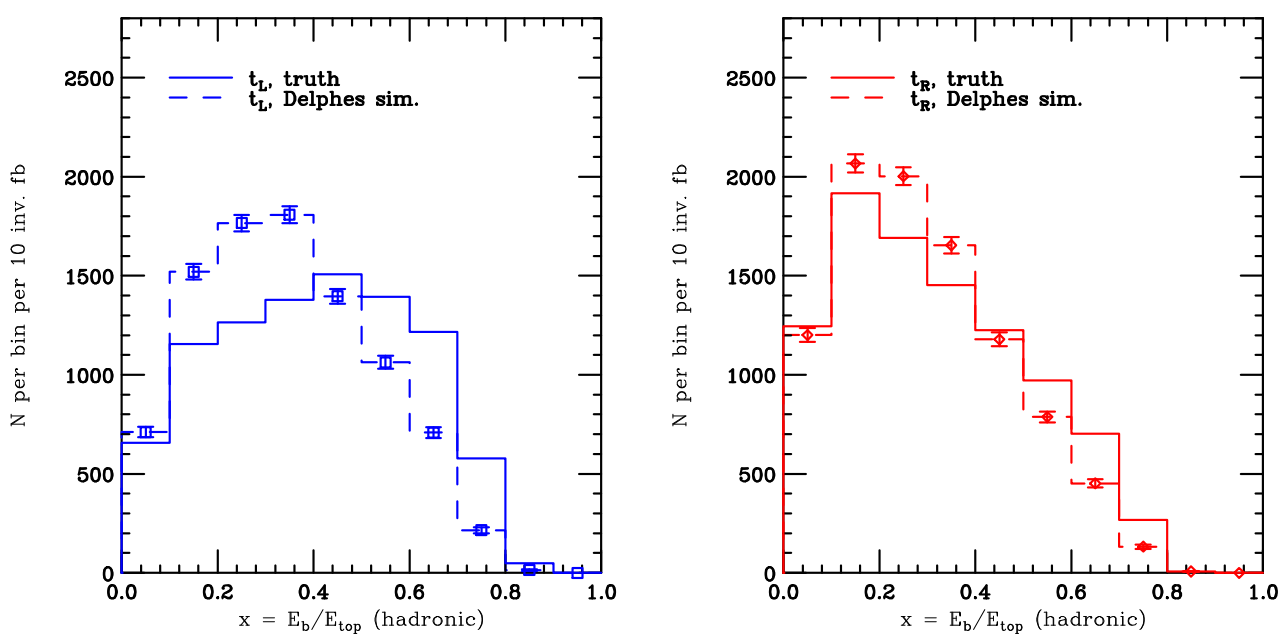

Figure 8. Shown in the figures is a comparison between the results obtained for the $x_{\text {top }}$ variable for the $1.5 \mathrm{TeV}$ flavour-changing $Z^{\prime}$ model before detector simulation (but applying all cuts and using equivalent jet-finding) and after the Delphes simulation for the left- and right- handed fermions (blue and red respectively).
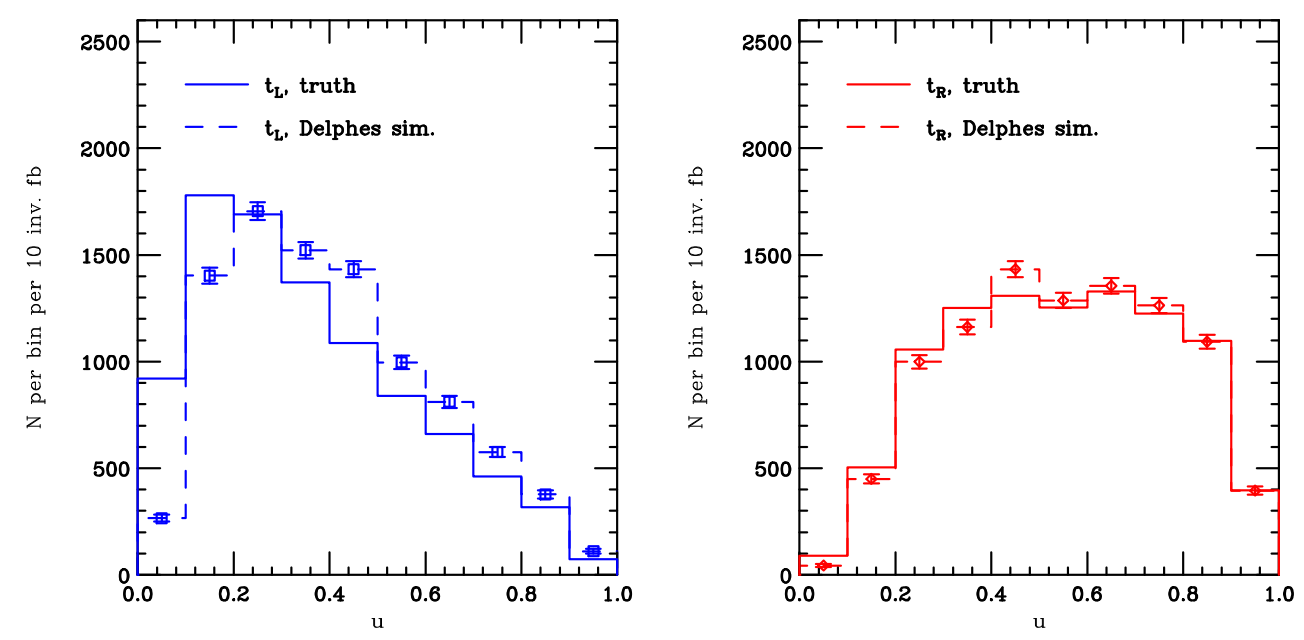

Figure 9. Shown in the figures is a comparison between the results obtained for the $u$ variable for the $1.5 \mathrm{TeV}$ flavour-changing $Z^{\prime}$ model before detector simulation (but applying all cuts and using equivalent jet-finding) and after the Delphes simulation for the left- and right- handed fermions (blue and red respectively).

no explicit reconstruction is required, the approximations that are associated with this process do not have a significant effect. As can be seen in the before/after plots in figures 8 and 9 , the $u$ variable is less sensitive to the experimental effects, which seem to 'squeeze' the distributions towards middle values of the energy fraction more dramatically in the case of $x_{\text {top }}$.

To investigate the effect of higher transverse momentum cuts on the objects used in calculating these variables, we constructed two further sets of plots with higher cuts which 

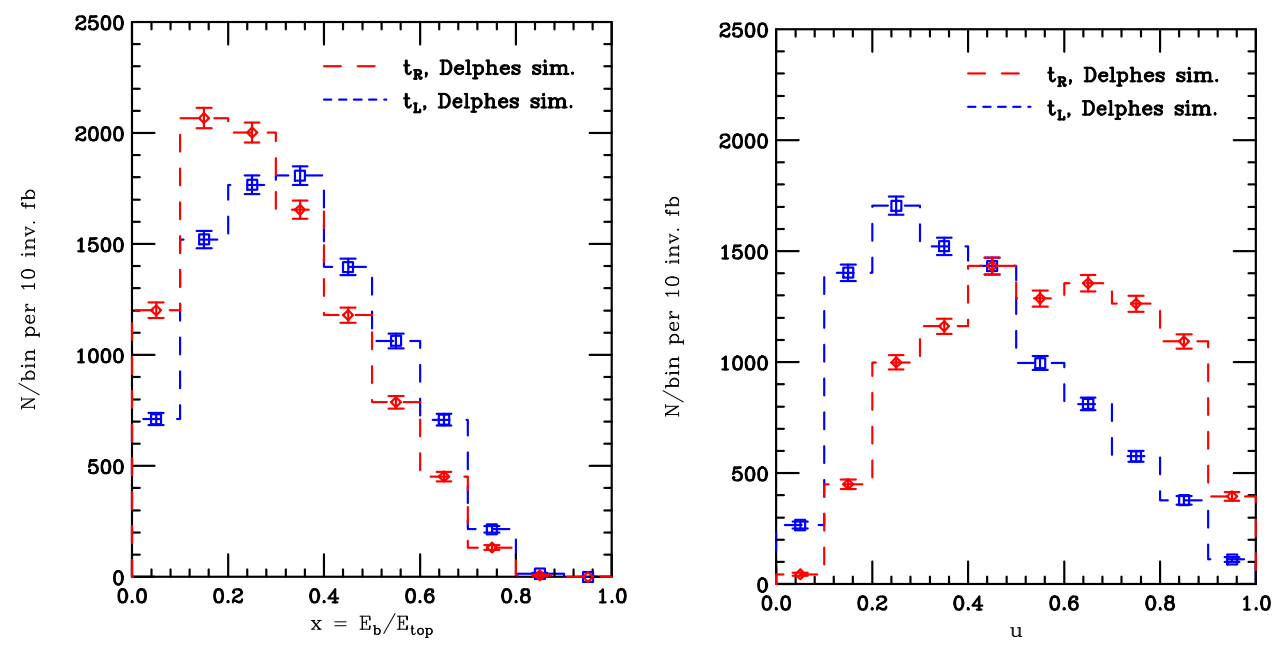

Figure 10. The $x_{\text {top }}$ (left panel) and $u$ (right panel) variables for left- or right-handed $Z^{\prime}$ bosons decaying to $u \bar{t}$ or $\bar{u} t$, obtained from the reconstructed events for an LHC run at $14 \mathrm{TeV}$, with $10 \mathrm{fb}^{-1}$.

\begin{tabular}{|c|c|c|}
\hline Cut set & $x_{\text {top }}$ & $u$ \\
\hline Min. & 40.7 & 145.8 \\
\hline A & 36.7 & 112.2 \\
\hline B & 37.6 & 75.8 \\
\hline
\end{tabular}

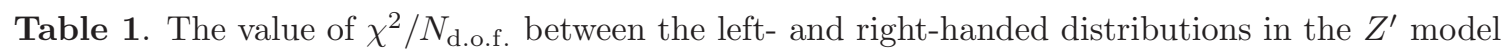
with decays to $t \bar{u}$ and $\bar{t} u$, for the three different sets of cuts. It is evident that the distributions are distinguishable even for the higher cuts, with the $u$ variable distribution performing best in all cases.

we call 'A' and 'B' and are, respectively, $p_{T}>30 \mathrm{GeV}$ and $p_{T}>50 \mathrm{GeV}$ for both jets and leptons. The resulting distributions are shown in figures 11 and 12 , where the set of cuts $\mathrm{A}$ is shown on the left, and set $\mathrm{B}$ on the right. Table 1 shows the value of $\chi^{2} / N_{\text {d.o.f. }}$ between the left- and right-handed distributions for the case of minimal cuts, as well as cuts ' $\mathrm{A}$ ' and 'B'. ${ }^{10}$ The value of $\chi^{2} / N_{\text {d.o.f. indicates how distinguishable the two distributions are }}$ statistically. It is evident that even in the case of higher cuts, discrimination in the specific scenario between the left- and right-handed modes is still possible. The higher cuts would also be beneficial for the rejection of proton-proton pile-up, ${ }^{11}$ which would be detrimental at high instantaneous luminosity.

${ }^{10}$ For the comparison of two binned data sets, $\chi^{2}$ can be defined as [24]:

$$
\chi^{2}=\sum_{i} \frac{\left(R_{i}-S_{i}\right)^{2}}{R_{i}+S_{i}},
$$

where $R_{i}$ and $S_{i}$ are the number of events in $i^{\text {th }}$ bin of the first and second data sets respectively.

${ }^{11}$ Pile-up is contamination originating from multiple secondary proton-proton collisions in the same bunch-crossing. 

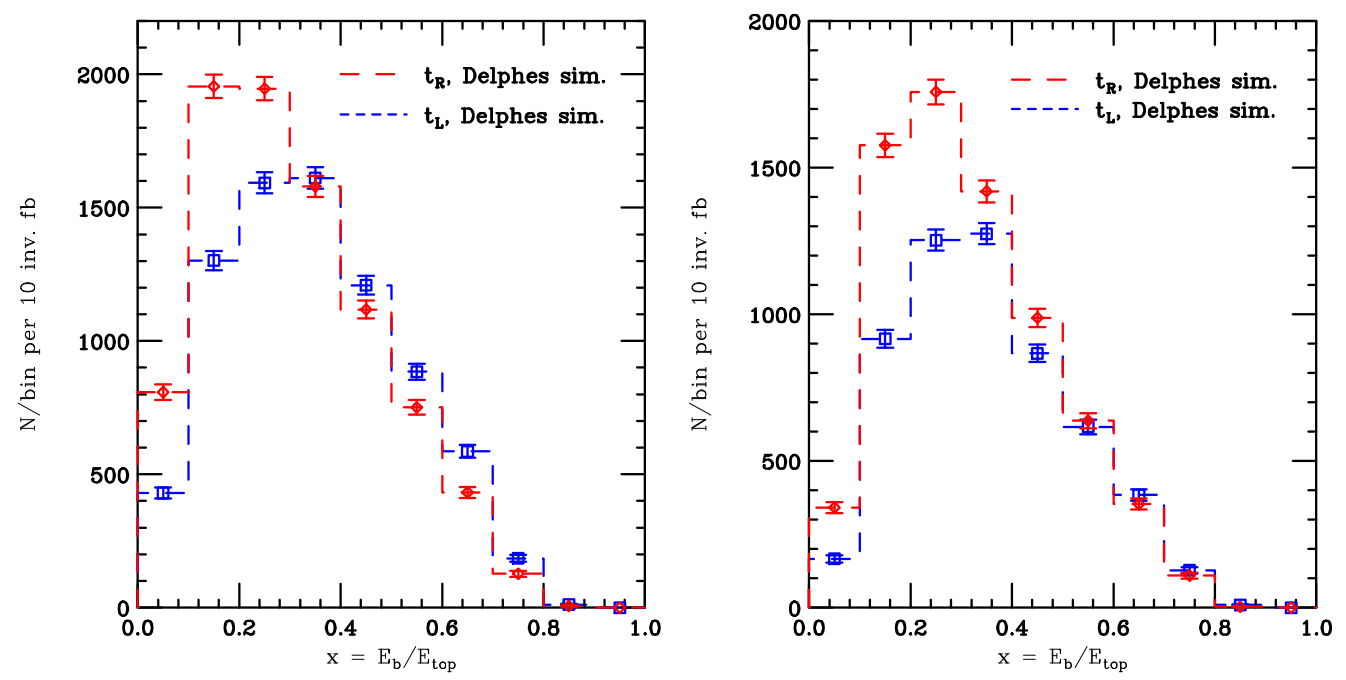

Figure 11. The $x_{\text {top }}$ variable for a left- or right-handed $Z^{\prime}$ bosons decaying to $u \bar{t}$ or $\bar{u} t$, obtained from the reconstructed events for an LHC run at $14 \mathrm{TeV}$, with $10 \mathrm{fb}^{-1}$, with the set of cuts A (left) and B (right), as explained in the text.
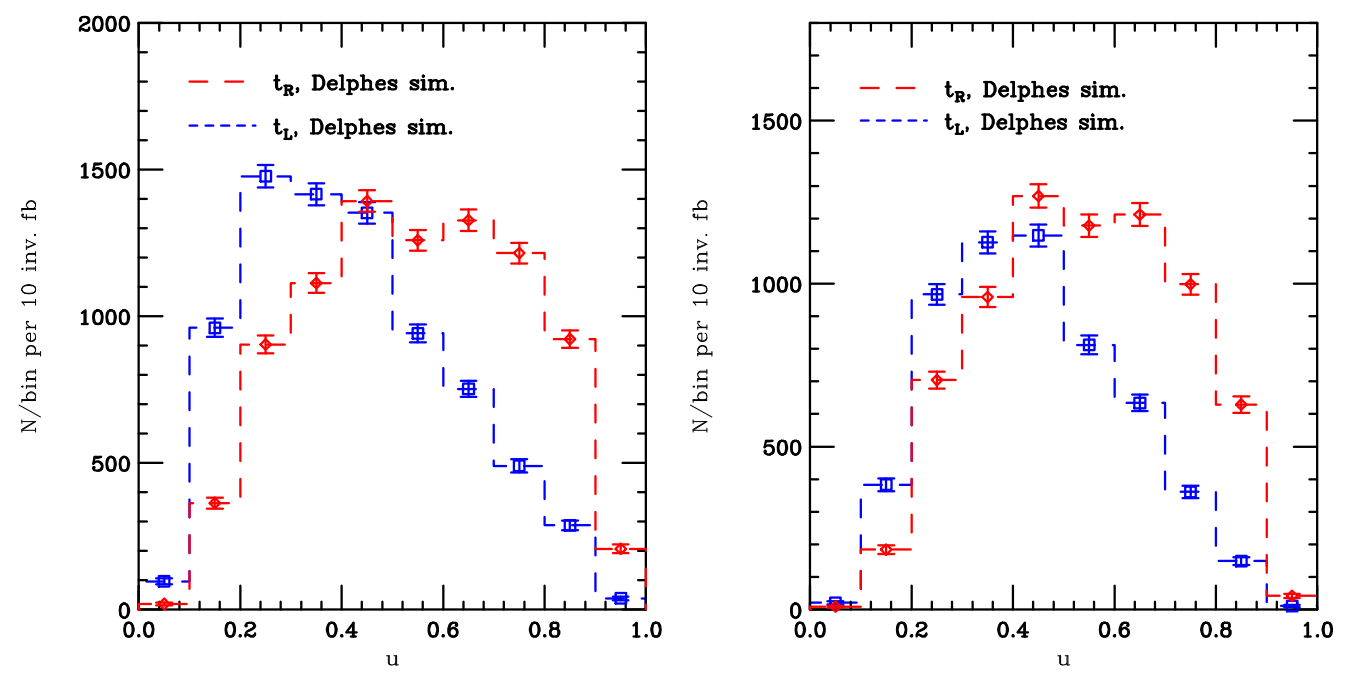

Figure 12. The $u$ variable for a left- or right-handed $Z^{\prime}$ bosons decaying to $u \bar{t}$ or $\bar{u} t$, obtained from the reconstructed events for an LHC run at $14 \mathrm{TeV}$, with $10 \mathrm{fb}^{-1}$, with the set of cuts A (left) and B (right), as explained in the text.

\section{$3.2 \quad Z^{\prime} \rightarrow \tau^{+} \tau^{-}$}

Another interesting example, which we can use to examine the effectiveness of the energy ratio $x_{\tau}$, is a model of a heavy $Z^{\prime}$ possessing a $\tau^{+} \tau^{-}$decay mode. For this model, we define a Lagrangian density similar to the one given in the previous section:

$$
\begin{aligned}
\mathcal{L}= & g_{Z^{\prime}}^{\tau \tau} Z^{\prime \mu} \bar{\tau}_{R} \gamma_{\mu} \tau_{R}+g_{Z^{\prime}}^{\tau \tau L} Z^{\prime \mu} \bar{\tau}_{L} \gamma_{\mu} \tau_{L} \\
& +g_{Z^{\prime}}^{u u R} Z^{\prime \mu} \bar{u}_{R} \gamma_{\mu} u_{R}+g_{Z^{\prime}}^{u u L} Z^{\prime \mu} \bar{u}_{L} \gamma_{\mu} u_{L}+\text { h.c. }
\end{aligned}
$$


We examine discrimination between two cases, $Z^{\prime} \rightarrow \tau_{R}^{+} \tau_{R}^{-}\left(g_{Z^{\prime}}^{\tau \tau R}=1, g_{Z^{\prime}}^{\tau \tau L}=0\right)$ and $Z^{\prime} \rightarrow \tau_{L}^{+} \tau_{L}^{-}\left(g_{Z^{\prime}}^{\tau \tau R}=0, g_{Z^{\prime}}^{\tau \tau L}=1\right)$, using the $x_{\tau}$ variable defined in eq. (2.2).

We checked that the irreducible backgrounds to the $\tau^{+} \tau^{-}$decay mode, e.g. originating from the $Z^{0} / \gamma \rightarrow \tau^{+} \tau^{-}$, can be rejected using an invariant mass cut on the $\tau$-jet pair. For example, an invariant mass cut of $\sim 500 \mathrm{GeV}$ offers a rejection factor of $\mathcal{O}\left(10^{-4}\right)$ for these backgrounds, bringing them to cross sections $\mathcal{O}(0.1 \mathrm{pb})$, while reducing the signal only by a factor of $\sim 0.7$ for the case of a $1.5 \mathrm{TeV} Z^{\prime}$, maintaining a cross section of a few picobarns. Possible backgrounds to the helicity determination may also arise from fake $\tau$-tagged QCD events, and these would need to be assessed by the individual experimental collaborations.

To compute the $x_{\tau}$ variable, one needs to reconstruct the neutrino energies from the $\tau$ decays. For this purpose, the authors of ref. [7] have used the collinear approximation to reconstruct the neutrino momenta. In the collinear approximation, the neutrinos are assumed to be collimated to the associated $\tau$-jets. This assumption is almost always good in cases of a heavy resonance decaying to $\tau$ leptons. Once the neutrino momentum directions are determined, the neutrino energies can be calculated using the missing transverse momentum constraint:

$$
\left(\begin{array}{l}
p_{\text {miss }}^{x} \\
p_{\text {miss }}^{y}
\end{array}\right)=\left(\begin{array}{cc}
\sin \theta_{\text {jet1 }} \cos \phi_{\text {jet } 1} & \sin \theta_{\text {jet2 }} \cos \phi_{\text {jet2 }} \\
\sin \theta_{\text {jet1 }} \sin \phi_{\text {jet } 1} & \sin \theta_{\text {jet2 }} \sin \phi_{\text {jet } 2}
\end{array}\right)\left(\begin{array}{c}
E_{\nu 1} \\
E_{\nu_{2}}
\end{array}\right),
$$

where $\theta_{\text {jeti }}, \phi_{\text {jeti }}$ are the polar and azimuthal angles respectively, related to jet $i, p_{\text {miss }}^{x, y}$ are the missing transverse momentum components and $E_{\nu i}$ is the energy of neutrino $i$.

However, when the two jets are back-to-back, i.e. $\phi_{\text {jet1 }}=\phi_{\text {jet } 2}+\pi$, the inverse of the matrix in eq. (3.7) becomes singular and any small mismeasurement on the missing transverse energy or jet momentum directions would cause a very large error on the reconstructed neutrino energy [25]. The back-to-back configuration is strongly preferable if a heavy resonance, such as the standard model Higgs boson or a $Z^{\prime}$, is considered.

One can avoid the use of the collinearity assumption by instead using information on the $\tau$ decay vertices [26]. The most useful and best-measured attribute of these is their impact parameter. The impact parameter $\mathbf{b}$ is the displacement of a decay vertex in a direction perpendicular to that of the visible decay momentum, in this case the $\tau$ jet momentum $\mathbf{p}_{j}$. Then the invisible momentum $\mathbf{p}_{\nu}$ must lie in the $\left(\mathbf{b}, \mathbf{p}_{j}\right)$ plane, so we can write $\mathbf{p}_{\nu}=x \mathbf{b}+y \mathbf{p}_{j}$. For hadronic $\tau$ decays, the invisible momenta are carried by single neutrinos and so their four-momenta are fixed by $x$ and $y$ for each decay. In this section we focus on hadronic $\tau$ decays, by including a lepton veto in our event selection criteria. These four quantities are subject to two linear missing- $p_{T}$ constraints and two quadratic $\tau$ mass-shell constraints, giving four (complex) solutions for the neutrino momenta which allow us to compute the invariant mass of the $\tau$ pair. In figure 13, we plot the real part of the invariant mass, where we use the true jets and missing transverse momenta for $10^{4}$ events. A distinct peak structure is seen at the input $Z^{\prime}$ mass of $1.5 \mathrm{TeV}$.

Unfortunately, our reconstruction method is still sensitive to the momentum mismeasurement. In figure 14, we show the hadronization and detector effects on the $Z^{\prime}$ mass reconstruction. In the left plot, the parton-level jets are replaced with the detector-level jets obtained from the Delphes simulation, in the middle plot only the parton-level missing 


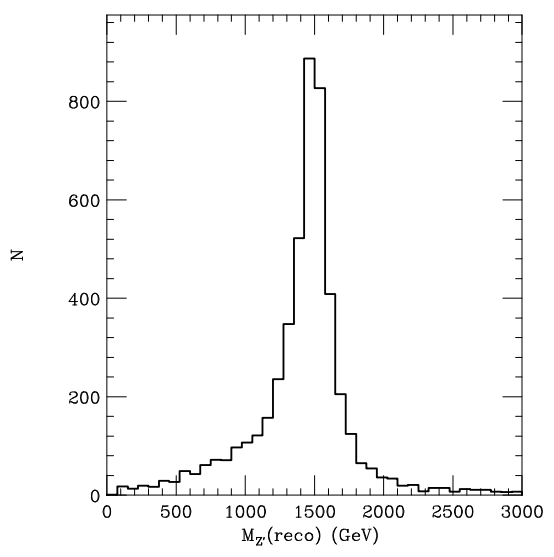

Figure 13. The real part of the complex solutions that arise after applying the two linear missing$p_{T}$ constraints and the two quadratic $\tau$ mass-shell constraints. A clear peak structure is seen at the input mass of $1.5 \mathrm{TeV}$.
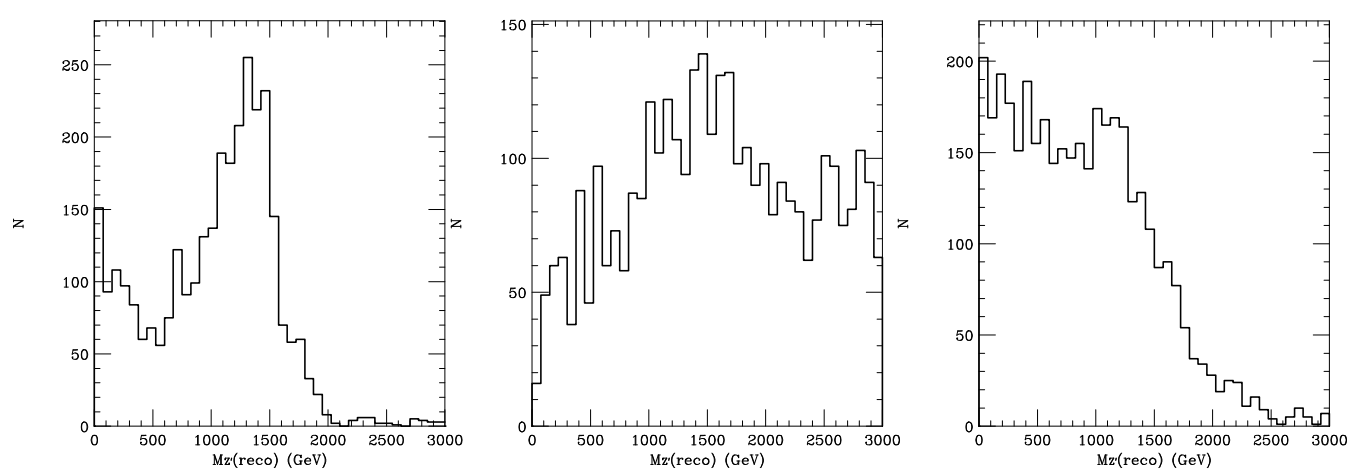

Figure 14. The distributions of the real part of the four complex solutions resulting from the reconstruction of the events after using detector-level jets instead of parton-level jets (left plot), detector-level missing transverse momentum instead of the true one (middle plot) and using completely detector-level objects (i.e. both for jets and MET, right plot). In all plots non-smeared $\tau$ vertices have been used.

transverse momentum is replaced with the detector-level one and in the right plot, we use all the detector-level objects. In all of the plots in figure 14, the true impact parameter was used. As can be seen, the peak structure is completely lost if one uses the detector objects. The event selection cuts here, and in all the results that follow in this section, are the default Delphes cuts for the ATLAS detector $\left(p_{T, \ell}>10 \mathrm{GeV}, p_{T, \text { jet }}>20 \mathrm{GeV}\right)$ along with the addition of the lepton veto (in this case rejecting all events with an identified lepton).

However it is important to realise that, in the model we are considering, the $Z^{\prime}$ mass can be measured independently from the decay modes containing the light quarks. In this case, we can use the mass shell constraint, $\left(p_{\tau_{1}}+p_{\tau_{2}}\right)^{2}=m_{Z^{\prime}}^{2}$, as an extra condition to correct for the detector resolution. To accomplish this we construct the following likelihood function:

$$
L_{\text {tot }}=L_{\text {detector }} \times L_{\text {phys }},
$$


where we have defined

$$
\begin{aligned}
L_{\text {detector }} & \equiv \Pi_{i=1}^{2}\left(P_{E_{i}} P_{\theta_{i}} P_{\phi_{i}}\right) \times P_{E}^{\text {miss }} \times P_{\phi}^{\text {miss }}, \\
L_{\text {phy }} & \equiv P_{m_{Z^{\prime}}} \times \Theta\left(\operatorname{Re}\left[E_{\nu_{1}}\right]\right) \times \Theta\left(\operatorname{Re}\left[E_{\nu_{2}}\right]\right) .
\end{aligned}
$$

The $P_{E_{i}}, P_{\theta_{i}}, P_{E}^{\text {miss }}, P_{\phi}^{\text {miss }}$ are Gaussian probability functions centred at the origin, with $\operatorname{arguments}\left(E_{j_{i}}-E_{j_{i}}^{\text {obs }}\right) / E_{j_{i}}^{\text {obs }},\left(\theta_{j_{i}}-\theta_{j_{i}}^{\text {obs }}\right),\left(p_{\text {Tmiss }}-p_{\text {Tmiss }}^{\text {obs }}\right) / p_{\text {Tmiss }}^{\text {obs }},\left(\phi_{p_{\text {Tmiss }}}-\phi_{p_{\text {Tmiss }}}^{\text {obs }}\right)$, respectively. We also use the flat probability distribution, $P_{\phi_{i}}$, for the $\left(\phi_{i}-\phi_{i}^{\text {obs }}\right)$ with a range [-0.9:0.9] so that the probability function matches the actual probability of mismeasurement, which is simulated by Delphes. The likelihood $L_{\text {phys }}$ allows us to correct the mismeasured observable by requiring physical conditions with some probability. $P_{m_{Z^{\prime}}}$ is a Gaussian probability function with an argument of $\left(m_{\tau \tau}-m_{Z^{\prime}}\right)$, where $m_{Z^{\prime}}$ is the true $Z^{\prime}$ mass assumed to be measured through some other decay mode. $\Theta(x)$ is 1 if $x>0$, 0 otherwise. The probability functions we use and the ones that appear in Delphes are shown in appendix B.

For each event, we generate 1000 pseudo-events, in which the observed momenta are slightly shifted in a random 'direction' according to the same probability function. We only keep the pseudo-event, $i_{\max }$ (corresponding to $L_{\mathrm{tot}}^{\max }$ ), that provides the maximum likelihood. We show the $\tau \tau$ invariant mass distribution obtained from $i_{\max }$ sample in figure 15.

In the left panel in figure 16, we show the relative difference between the true neutrino energy and the reconstructed neutrino energy by the likelihood method. As can be seen, the true neutrino energy is well reconstructed on an event-by-event basis with about $50 \%$ error. The right panel in figure 16 shows the reconstructed $x_{\tau}$ variable in this method, using $1 \mathrm{fb}^{-1}$ of integrated luminosity at a $14 \mathrm{TeV}$ LHC (corresponding to $\sim 7000$ events before cuts). The red (blue) solid histogram is obtained from $Z^{\prime} \rightarrow \tau_{R}^{+} \tau_{R}^{-}\left(Z^{\prime} \rightarrow \tau_{L}^{+} \tau_{L}^{-}\right)$sample. The lepton veto in this realistic case was applied by requiring no leptons with $p_{T}>10 \mathrm{GeV}$ in $|\eta|<2.4$. The dashed histogram is the corresponding parton-level distribution of $x_{\tau}$. It is obvious that the reconstructed $x_{\tau}$ has a very similar distribution to the parton-level one, and the difference between the left and right-handed $x_{\tau}$ distributions is visible even after the effects of detector resolution. The value of $\chi^{2} / N_{\text {d.o.f }}$ was found to be $\sim 13.8$, indicating the high difference between the left- and right-handed $x_{\tau}$ histograms.

\subsection{Third-generation leptoquark pair-production}

Methods for reconstructing third-generation scalar leptoquark states in events where they were pair-produced have been studied in ref. [13]. There, different mass variables were constructed and all the possible combinations of decay modes were studied, including QCD and detector effects. The main background, $t \bar{t}$ production, was shown in ref. [13] to produce negligible contribution in the suggested reconstruction variables in the vicinity of the leptoquark signal. We will focus here on the leptoquark types that can decay to a top quark and a tau lepton.

For a list of states that can decay to the $t \tau$ modes see tables 1 and 2 in ref. [13]. Instead of focusing on a specific leptoquark type, we consider a general scalar leptoquark which 


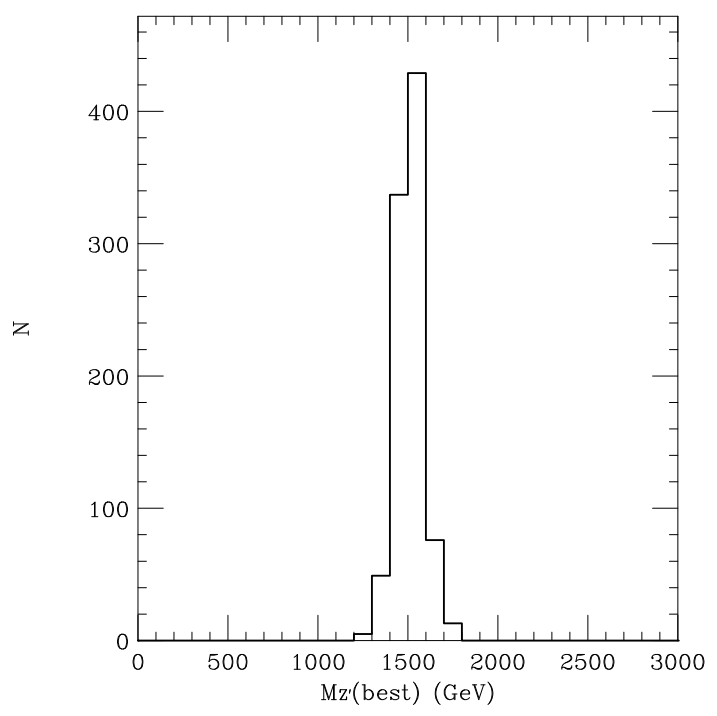

Figure 15. The $\tau \tau$ invariant mass of the maximum likelihood sample as described in the text. The distribution is sharply peaked at the input $Z^{\prime}$ mass of $1.5 \mathrm{TeV}$.
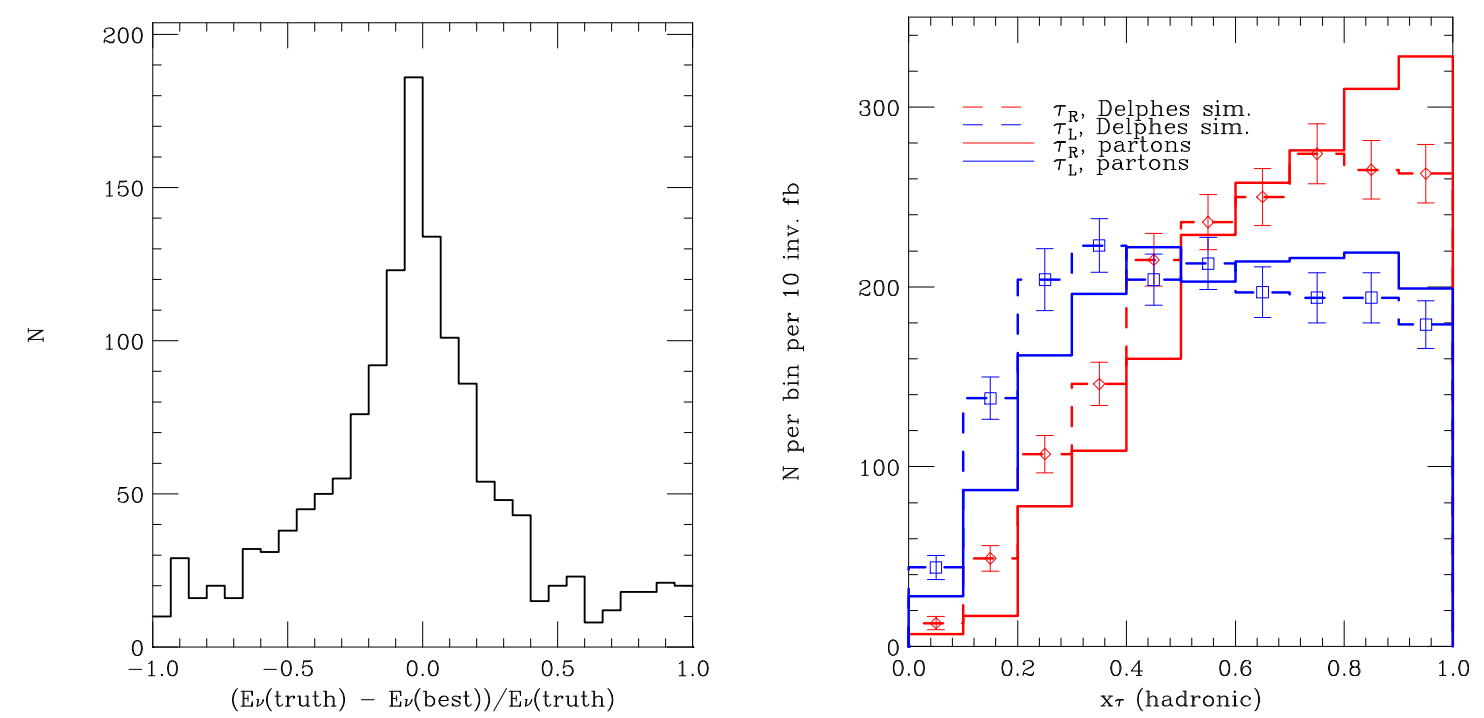

Figure 16. The relative difference between the true neutrino energy and the reconstructed neutrino energy using the likelihood method described in the text (left panel) and the resulting energy fraction, $x_{\tau}$ distributions for the purely left- and purely right-handed cases (right panel) for $1 \mathrm{fb}^{-1}$ of integrated luminosity at $14 \mathrm{TeV}$.

possesses a branching fraction of 1 to a top quark and a $\tau$ lepton. We call the leptoquarks of this type, with electromagnetic charge $\pm 5 / 3, S_{L L}$ and $S_{R R}$, where the index indicates that the leptoquark will decay either to $t_{L} \tau_{L}$ or $t_{R} \tau_{R}$ respectively. In appendix $\mathrm{C}$ we consider leptoquarks of electromagnetic charge $\pm 1 / 3$ decaying to the mixed combinations $\bar{t}_{R} \tau_{L}$ or $\bar{t}_{L} \tau_{R}\left(S_{R L}\right.$ and $S_{L R}$ respectively). To obtain results for other scenarios of leptoquarks that decay into this mode, one has to simply rescale the results to account for the appropriate cross sections. 

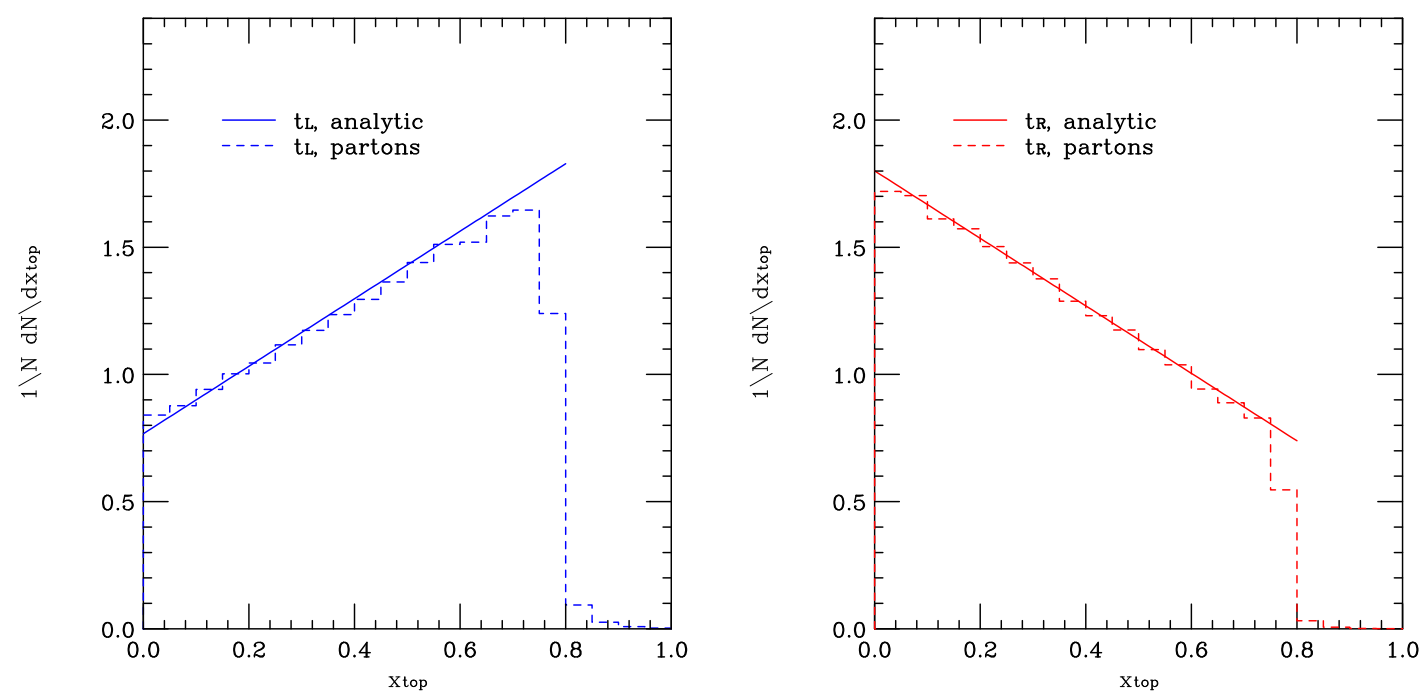

Figure 17. The Monte Carlo results for the energy fractions $x_{\text {top }}=\mathcal{E}_{b} / \mathcal{E}_{\text {top }}$ for $\tau_{L} t_{L}$ (left) and $\tau_{R} t_{R}$ (right) in the highly-boosted case. These are compared to analytical predictions as described in the text.

\subsubsection{Parton-level results}

We produce Monte Carlo distributions of the variables outlined in section 2 for leptoquark states to compare to the predicted distributions at parton level. We do not present partonlevel distributions for the $\tau$, as the corresponding parton-level results have been already extracted from the Monte Carlo event generator itself and appear in figure 3.

The $S_{X X}(X \in\{R, L\})$ leptoquark can decay to $t \tau$ modes, described by the Lagrangian terms:

$$
g_{R R} \bar{t}_{R}^{c} \tau_{R} S_{R R}+g_{L L} \bar{t}_{L}^{c} \tau_{L} S_{L L}+\text { h.c. . }
$$

In the present study we set either $g_{R R}=0, g_{L L}=1$ (which we call purely left-handed) or vice versa: $g_{R R}=1, g_{L L}=0$ (which we call purely right-handed). The result for the highlyboosted $x_{\text {top }}$ distributions obtained for purely left- and right-handed events in HERWIG++ is shown in figure 17 with the appropriate analytic prediction. The proton-proton centreof-mass energy was set to $140 \mathrm{TeV}$ and the leptoquark mass was set to $20 \mathrm{TeV}$, so that the top quarks are well within the highly-boosted region.

We also present the top quark results originating from the decay of scalar leptoquarks of mass $400 \mathrm{GeV}$ at the LHC with $p p$ centre-of-mass energy of $14 \mathrm{TeV}$. Figures 18 and 19 show the left- and right-handed distributions of the variables $x_{\text {top }}$ and $u$ respectively, produced using the HERWIG++ Monte Carlo event generator and including semi-analytical predictions. These include the effects of the finite top and tau masses, which introduce a mixture of helicities even though the Lagrangian terms are purely chiral, and the effect of the variation of the top boost in the lab frame, $\beta_{t}$.

The semi-analytical predictions have been produced using Monte Carlo techniques as described in section 3.1: the $x_{\text {top }}$ distribution was produced by distributing $\cos \theta_{b}$ according to $P\left(\cos \theta_{b}\right)=\left(1+P_{t} k_{b} \cos \theta_{b}\right)$, and the $\beta_{t}$ distribution for the top quark boost was extracted 

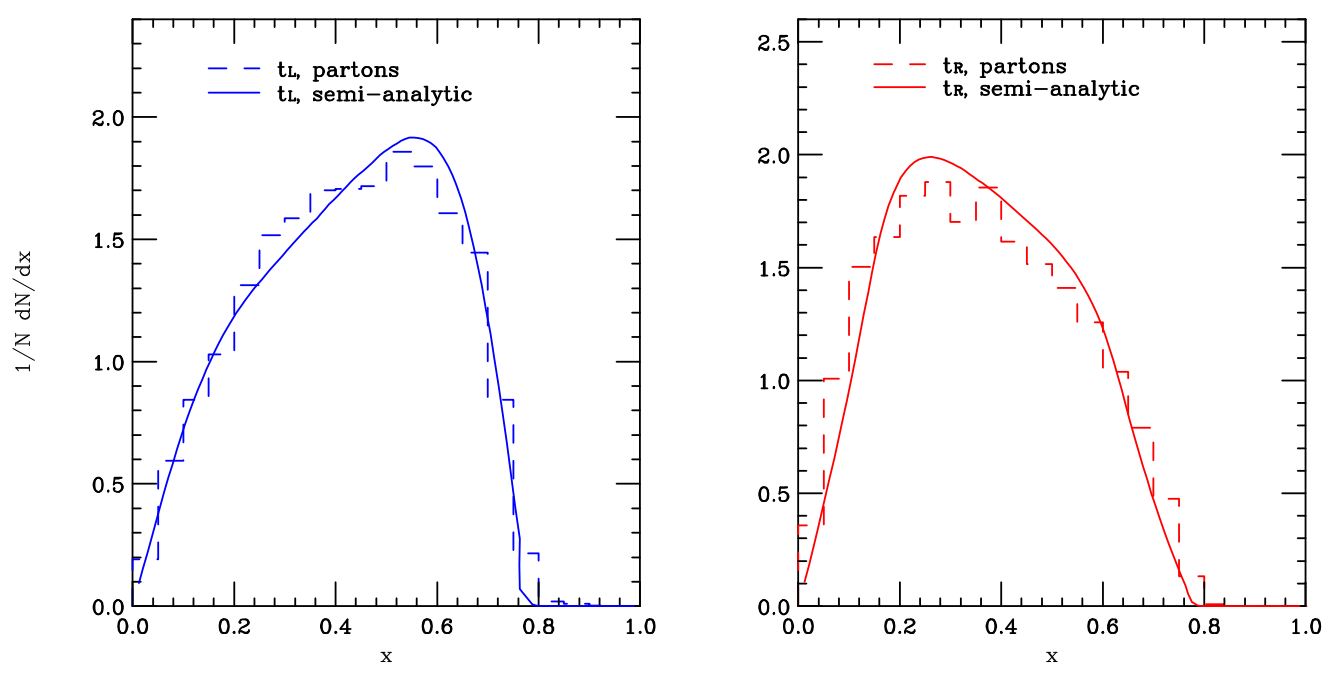

Figure 18. The results for the energy fractions $x_{\text {top }}=\mathcal{E}_{b} / \mathcal{E}_{\text {top }}$ for $\tau_{L} t_{L}$ (left) and $\tau_{R} t_{R}$ (right) for a $400 \mathrm{GeV}$ mass at $14 \mathrm{TeV} p p$ centre-of-mass energy. These are compared to semi-analytical predictions as described in the text.
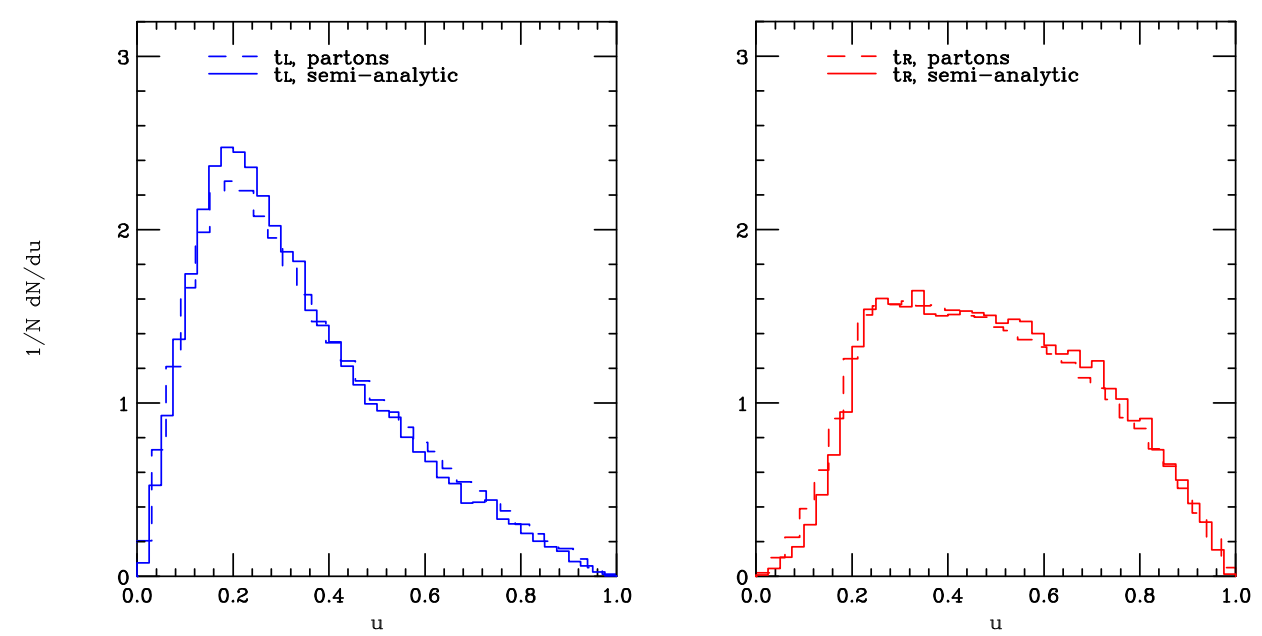

Figure 19. The Monte Carlo results for the energy fractions $u=\frac{\mathcal{E}_{\ell}}{\mathcal{E}_{\ell}+\mathcal{E}_{b}}$ for $\tau_{L} t_{L}$ (left) and $\tau_{R} t_{R}$ (right) for a $400 \mathrm{GeV}$ mass at $14 \mathrm{TeV}$ pp $\mathrm{COM}$ energy. These are compared to semi-analytical predictions as described in the text.

from the Monte Carlo event generator (see appendix E.2). Using either a fit or the extracted distribution yields indistinguishable results.

Since the boost of the parent leptoquark in the lab frame and the daughter top quark in the lab frame are correlated, we extracted the two-dimensional distribution $P\left(\beta_{p}, \beta_{t}\right)$ from the HeRwIG++ event generator (appendix E.2). In principle, the $\beta_{p}$ distribution can be calculated for any process using the hadron parton density functions and an assumption for the hard process, in this case scalar $\mathrm{SU}(3)_{c}$ triplet pair-production. The variable $\beta_{p}$ in the case of pair-produced leptoquarks of mass $M_{L Q}$ is then related to the centre-of-mass energy 
$Q$ by $\beta_{p}=\sqrt{1-4 M_{L Q}^{2} / Q^{2}}$. The method described in ref. [4] was then used to calculate the detected polarisation of the top for each event. The effect reduces the polarisation on average by less than $10 \%$. The mixing of helicities due to the mass of the top quark is low (less than $0.1 \%$ ) due to the fact that it is produced along with a very light fermion (the $\tau$ lepton) in the case we are considering.

The $u$ distribution was produced in a similar way, using the full polarised top matrix element (see appendix D). The $W$ decay to a lepton and a neutrino was set up in the $W$ rest frame using a polar angle $\bar{\theta}$ and an azimuthal angle $\bar{\phi}$ for the lepton and neutrino momenta. These were then boosted to the top frame, where the $b$ quark and $W$ boson momenta were distributed in the top frame using a single polar angle $\tilde{\theta} .^{12}$ The $W$ mass was distributed according to a Breit-Wigner, centred about $m_{W}$. The effect of the $W$ width was found to be small. The distribution was then calculated by taking the ratio:

$$
u=\frac{E_{\ell}+\beta_{t} p_{\ell}^{z}}{E_{\ell}+\beta_{t} p_{\ell}^{z}+E_{b}+\beta_{t} p_{b}^{z}}
$$

where $E_{\ell}$ and $E_{b}$ are the lepton and $b$-quark energies in the top rest frame and $\beta_{t}$ is again the boost of the top, sampled from either from the fit (eq. (E.21)) or the Monte Carlo distribution directly. The calculations of the effect of the finite top and $\tau$ masses in the decay of a scalar and the relation of the top axis of polarisation and direction of motion follow those which appear in ref. [4] and are described briefly in appendix E.

\subsubsection{Simulation and reconstruction}

A mass reconstruction strategy for the $(t \tau)(t \tau)$ decay mode is described in ref. [13]. The reconstruction there focuses on the modes $\bar{S}(S) \rightarrow b j(j) j_{1} \nu_{1}, S(\bar{S}) \rightarrow b \ell \nu_{3} j_{2} \nu_{2}$. We call this the hadronic/semi-leptonic mode, as opposed to the fully hadronic mode which we will examine below. An important assumption, that we have already discussed in section 3.2, which allowed for the full reconstruction of this decay mode, is the collinearity of the decay products of the tau leptons, owing to the fact that they are highly-boosted in the lab frame. This has been tested for different leptoquark masses in ref. [13]. The assumption can be applied by the relation $p_{\tau_{i}}=z_{i} p_{j_{i}}$, where $i=1,2$ and the energy ratios imply that $z_{i} \geq 1 .{ }^{13}$ In ref. [13] a quartic equation was obtained for the energy ratio $z_{2}$ and each solution is an unique reconstruction of the whole event. This method provides a clean way to discriminate the leptoquark signal from the background. However, our preliminary attempts to reconstruct the helicity of this mode have indicated that both the number of events, and the quality of the individual four-momenta reconstruction are insufficient for detailed determination of the top or $\tau$ helicities. Hence, we focus instead on the topology that contains two fully hadronic tops, shown in figure 20 .

\footnotetext{
${ }^{12}$ Initially there are 9 degrees of freedom coming from the momenta of the $b, \ell$ and $\nu$. Four-momentum conservation offers four constraints and the mass-shell conditions for the top and $W$ offer a further two. This leaves us with the three degrees of freedom: $\bar{\theta}, \bar{\phi}$ and $\tilde{\theta}$.

${ }^{13}$ Since the events we are considering are not a $\tau \tau$ resonance, there is no issue with back-to-back $\tau$ leptons as the one which previously appeared in section 3.2 .
} 


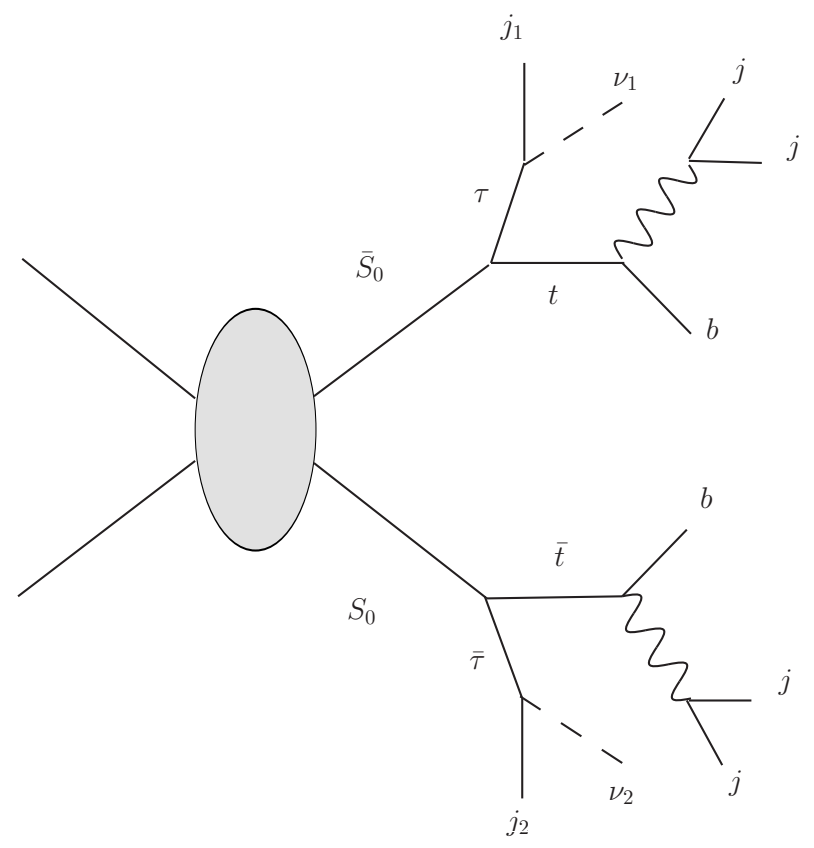

Figure 20. Pair-production of a leptoquark pair with decay to $(t \tau)(t \tau)$, followed by two fully hadronic top decays.

The signal was generated using the HERWIG++ event generator, including initial- and final-state radiation (ISR and FSR), hadronization effects and the underlying event (multiple parton interactions) and the detector response was simulated using the Delphes package with the default ATLAS settings, modified by the $b$-tagging function of eq. (3.5), without the trigger simulation. Here, we also used the anti- $k_{T}$ algorithm with radius parameter $R=0.4$. The following cuts were applied on data corresponding to an integrated luminosity of $100 \mathrm{fb}^{-1}$ at $14 \mathrm{TeV}$ :

- A minimum of 6 jets (since the jets originating from the $W$ could be identified as one jet in the cases where the $W$ is highly boosted).

- The missing transverse momentum in the event, $\mathbb{E}_{T}>20 \mathrm{GeV}$.

- Two $\tau$-tagged jets and two $b$-tagged jets, all with the extra requirement that they have $p_{T}>20 \mathrm{GeV}$.

Note that these are the minimal cuts that one could impose in principle experimentally, and are lower than those imposed in the reconstruction techniques proposed in ref. [13].

Full reconstruction of the decay topology shown in figure 20 is possible, since we would only be missing the neutrino momenta originating from the decays of the highly-boosted $\tau$ leptons, once the hadronic tops have been reconstructed. Using the approximation for the tau collinearity, one is left with only two unknowns, the $z_{1}$ and $z_{2}$ energy fractions. By assuming that the $x$ and $y$ components of the missing momentum are equal to those of the 
sum of the two neutrino components, we obtain two linear equations:

$$
\begin{aligned}
& p_{\text {miss }}^{x}=p_{j 1}^{x}\left(z_{1}-1\right)+p_{j 2}^{x}\left(z_{2}-1\right), \\
& p_{\text {miss }}^{y}=p_{j 1}^{y}\left(z_{1}-1\right)+p_{j 2}^{y}\left(z_{2}-1\right),
\end{aligned}
$$

which can be solved to give:

$$
\begin{aligned}
& z_{1}=1+\frac{p_{j 2}^{y} p_{\mathrm{miss}}^{x}-p_{j 2}^{x} p_{\mathrm{miss}}^{y}}{p_{j 1}^{x} p_{j 2}^{y}-p_{j 2}^{y} p_{j 2}^{x}}, \\
& z_{2}=1-\frac{p_{j 1}^{y} p_{\mathrm{miss}}^{x}-p_{j 1}^{x} p_{\mathrm{miss}}^{y}}{p_{j 1}^{x} p_{j 2}^{y}-p_{j 2}^{y} p_{j 2}^{x}}
\end{aligned}
$$

The invariant mass of each of the two leptoquarks may be written as $m_{S}^{2}=\left(p_{t}+p_{\tau}\right)^{2}$, resulting in the following expression:

$$
m_{S}^{2}=2 z_{i} p_{t i} \cdot p_{j i}+m_{\text {top }}^{2},
$$

where we have neglected the $\tau$ mass term. Using eqs. (3.13), we obtain two values of $m_{S}$ per event. Since this analysis would be performed after potential discovery, we would already have a measurement of the mass of the leptoquark. This would allow for elimination of backgrounds that may contribute and alter the energy fraction distributions.

To assess the possibility of measuring the helicity of the top quarks and tau leptons, we generated $100 \mathrm{fb}^{-1}$ of a fully hadronic sample for purely left-handed or right-handed couplings and passed them through the Delphes simulation. We then analysed events which contained $2 \tau$-tagged jets and $2 b$-tagged jets. We looked for 1 or 2 jets which reconstructed the top mass in conjunction with the tops, within an $80 \mathrm{GeV}$ window. ${ }^{14}$ For completeness, we show in figure 21 the resulting reconstructed masses using the above method, without any attempt to optimise the resulting values. In the figure, we plot all the 4 entries per event, which are a consequence of the two leptoquark masses $\left(m_{S}, m_{\bar{S}}\right)$ and the two-fold combinatoric ambiguity, arising from the possible pairings of the top quarks and the $\tau$ leptons. To obtain the best value of the variables $x_{\text {top }}$ and $x_{\tau}$, we choose the combination which yields the best leptoquark mass, that is, the one closest to the true mass. We then obtain two values of the $x_{\tau}$ variable: $x_{\tau, 1 / 2}=1 / z_{1 / 2}$ and two values of the $x_{\text {top }}$ variable by using the energies of the two $b$-jets and the reconstructed top energy. A comparison between the results obtained before detector simulation, but applying all cuts and using equivalent jet-finding, and after the Delphes simulation, is shown in figures 22 and 23 on the left- and right- handed fermions, for the variables $x_{\text {top }}$ and $x_{\tau}$. The results without detector simulation have been normalised to the number of events resulting after Delphes simulation. The differences that arise at low and high energy fractions and can be attributed primarily to the efficiency of the tagging algorithms and the overall differences to the smearing of the four-momenta due to the simulation of the response of the detector. Comparisons of the Delphes results are shown in figure 24 for the purely left- and right-handed cases.

\footnotetext{
${ }^{14}$ To further improve the 'top-tagging' capabilities of the analysis, one can employ a more advanced tagging algorithm such as the one presented in ref. [27]. For our purposes, the simpler reconstruction method of requiring combinations of jets to satisfy the top mass is sufficient to provide good results.
} 


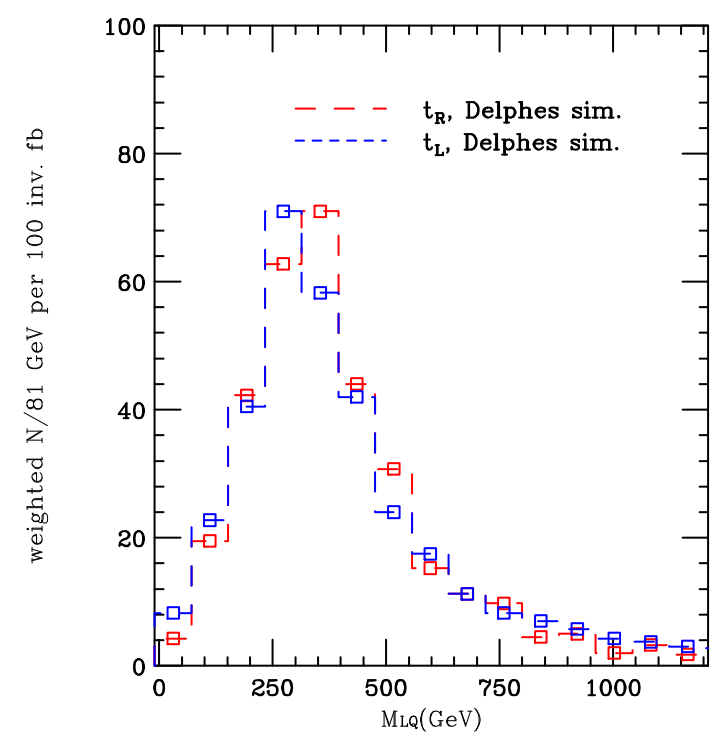

Figure 21. Mass reconstruction in the fully hadronic mode for the purely left- and right-handed cases for an integrated luminosity of $100 \mathrm{fb}^{-1}$. There are 4 entries per event corresponding.
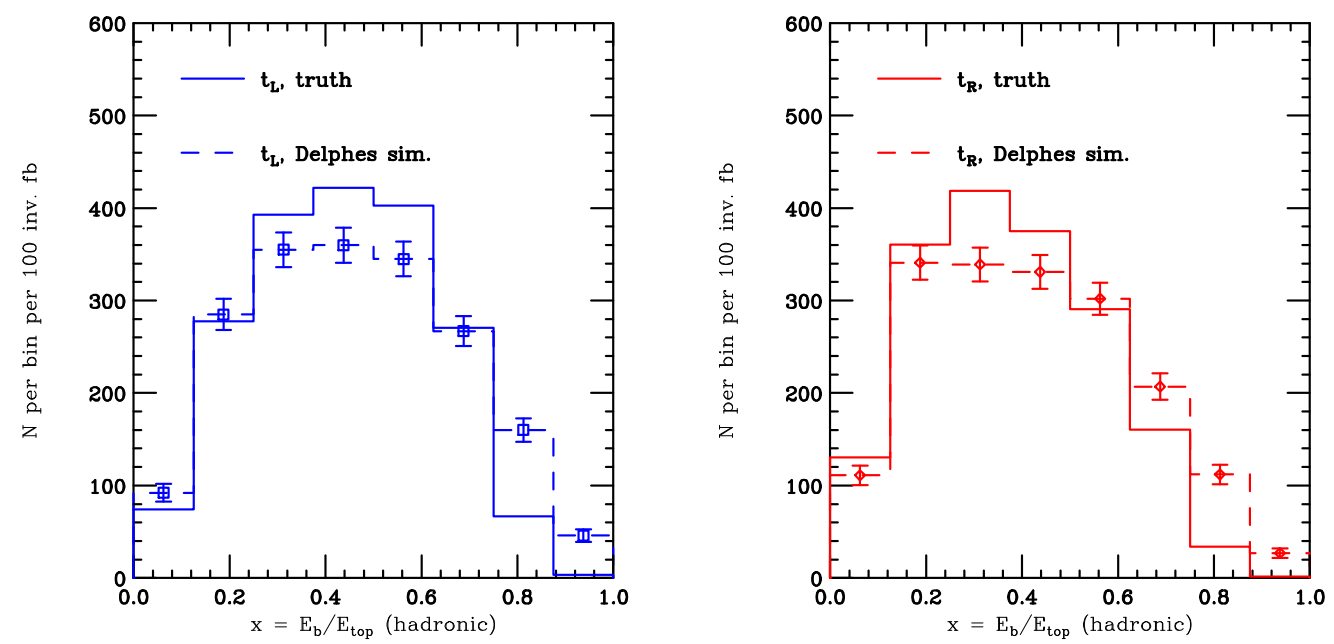

Figure 22. Shown in the figures is a comparison between the results obtained for the $x_{\text {top }}$ variable for $400 \mathrm{GeV}$ leptoquarks before detector simulation (but applying all cuts and using equivalent jet-finding) and after the Delphes simulation for the left- and right- handed fermions (blue and red respectively).

To assess the discrimination capabilities of the distributions, we calculated the $\chi^{2} / N_{\text {d.o.f. }}$ between the left- and right-handed distributions. To investigate the effect of higher transverse momentum cuts, we re-ran the analysis with two higher $p_{T}$ cuts on the jets and the missing transverse momentum: set 'A-prime' with $p_{T}$ and $\mathbb{E}_{T}>25 \mathrm{GeV}$ and set 'A' with $p_{T}$ and $\mathbb{E}_{T}>30 \mathrm{GeV}$. The results are shown in table 2 . 

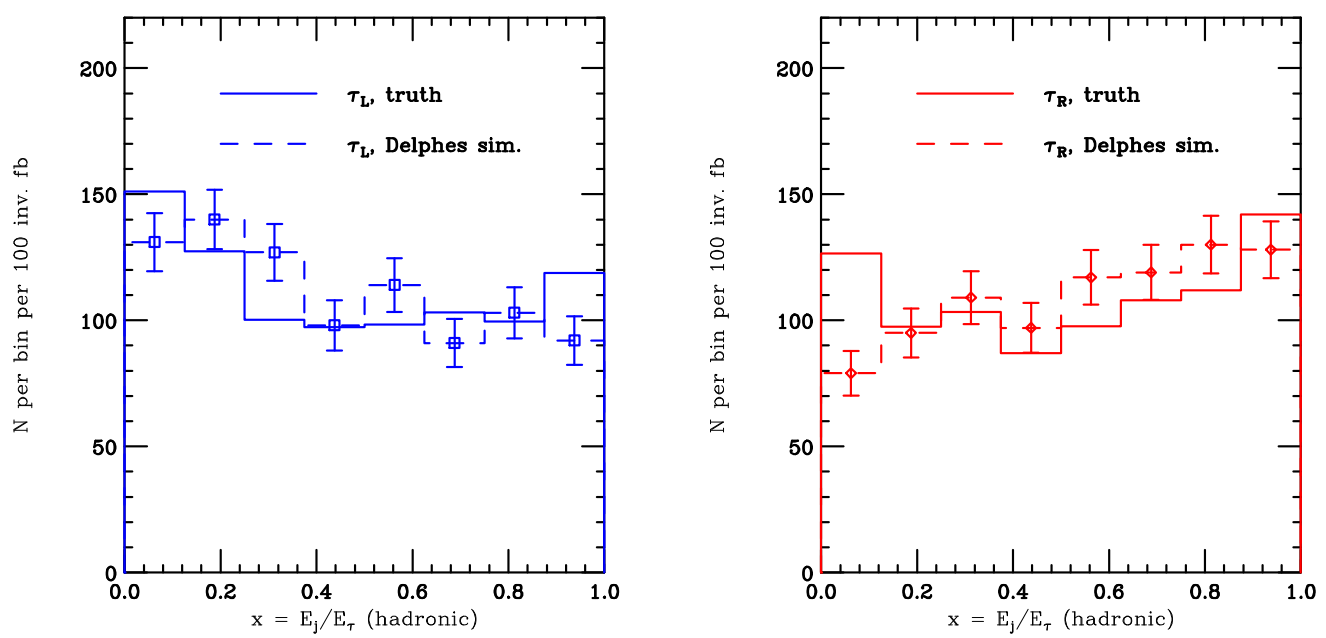

Figure 23. Shown in the figures is a comparison between the results obtained for the $x_{\tau}$ variable for $400 \mathrm{GeV}$ leptoquarks before detector simulation (but applying all cuts and using equivalent jetfinding) and after the Delphes simulation for the left- and right- handed fermions (blue and red respectively).
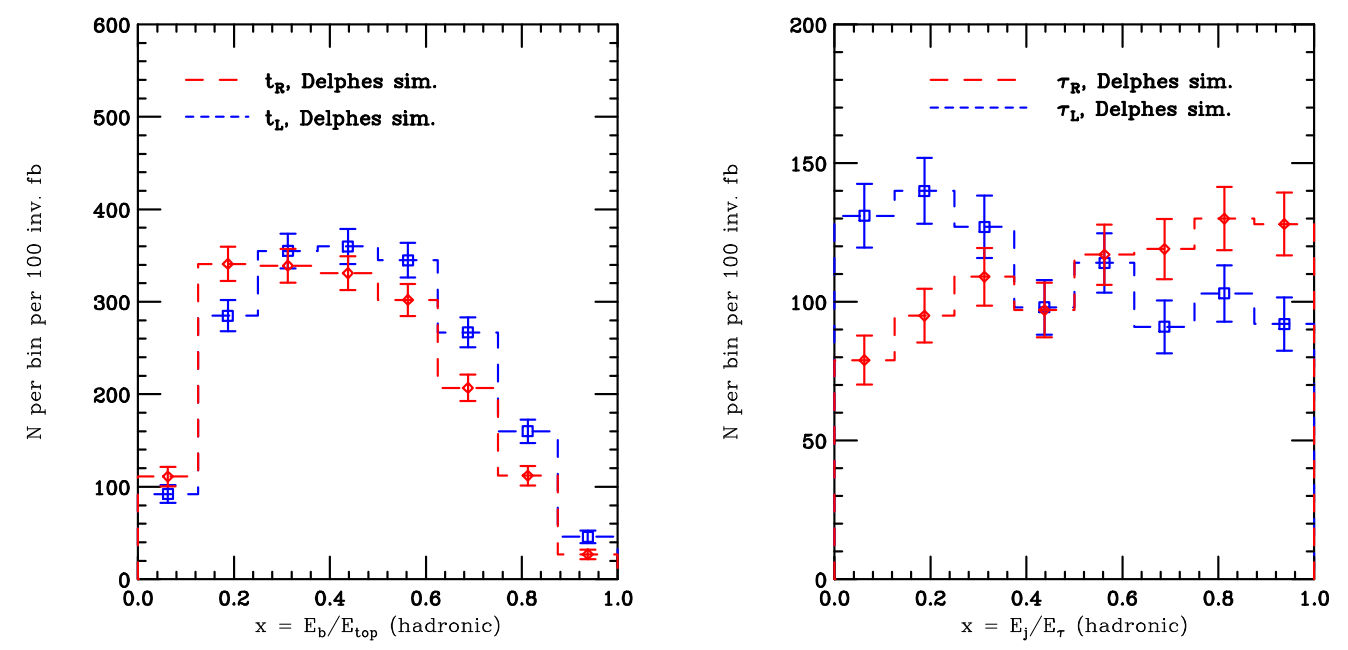

Figure 24. The $x_{\text {top }}$ (left panel) and the $x_{\tau}$ (right panel) variables for left- or right-handed $t \tau$ for an integrated luminosity of $100 \mathrm{fb}^{-1}$ modes originating from the reconstructed events in the fully hadronic case, using the method described in the text.

\section{Conclusions}

We have investigated variables that have been defined for the purpose of determining the helicity of top quarks and tau leptons, in a more realistic setting than what has been done thus far in the literature. We first examined these analytically in the highly-boosted case, where the calculation is simplified, and no explicit event reconstruction is required for the $u$ variable. Subsequently we focused on two specific models: one containing a 


\begin{tabular}{|c|c|c|}
\hline Cut set & $x_{\text {top }}$ & $x_{\tau}$ \\
\hline Min. & 4.0 & 4.5 \\
\hline A-prime & 2.9 & 3.8 \\
\hline A & 1.9 & 2.8 \\
\hline
\end{tabular}

Table 2. The value of $\chi^{2} / N_{\text {d.o.f. }}$ between the left- and right-handed distributions in the leptoquark $t \tau$ decay scenario, for the three different sets of cuts. It is evident that the distributions become more difficult to distinguish for the higher cuts at the given integrated luminosity of $100 \mathrm{fb}^{-1}$.

new heavy vector boson, $Z^{\prime}$, with decays to either a light jet and a top quark or two taus, and a specific scenario in scalar leptoquark pair-production, in which the decay of both leptoquarks is into a top quark and a $\tau$ lepton. We examined the flavour-changing $Z^{\prime}$ model at parton level, producing the relevant distributions semi-analytically and comparing these directly to the Monte Carlo-generated distributions. We considered experimental and reconstruction effects for the case of a LHC at proton-proton centre-of-mass energy of $14 \mathrm{TeV}$ and $10 \mathrm{fb}^{-1}$ of integrated luminosity, and we investigated the applicability of the helicity discrimination variables. We found that in the case of a $1.5 \mathrm{TeV} Z$ ' that can decay into an up quark and a top quark, the top quark helicity can be determined, even for higher momentum cuts. For the $Z^{\prime}$ model with decays to $\tau^{+} \tau^{-}$we used the $\tau$ decay vertex information along with a likelihood method to correct for detector resolution effects, resulting in good discrimination between the left- and right-handed modes for $1 \mathrm{fb}^{-1}$ of integrated luminosity. For the leptoquark pair-production model, for $400 \mathrm{GeV}$ leptoquarks decaying into a top quark and a $\tau$ lepton each, we examined reconstruction of the case when both the decaying fully hadronically. Discrimination in this scenario is more challenging, but values of $\chi^{2} / N_{\text {d.o.f. }} \sim 2-3$ can be obtained even with higher than minimal cuts for an integrated luminosity of $100 \mathrm{fb}^{-1}$.

To summarise, we have assessed the magnitude of the effects of QCD, cuts on the transverse momentum, detector effects and finally the reconstruction issues that arise in this phenomenological study of helicity variables. This work is indicative of the difficulties that arise in 'measuring' the helicities to determine the form of the interactions of new particles to quarks and leptons of the third generation. To fully determine the potential performance of these variables, the next step would be for the experimental collaborations to perform similar analyses, with realistic detector response and particle identification techniques.

\section{Acknowledgments}

We would like to thank the Cavendish Laboratory's High Energy Physics group for allowing continuous use of their computing facilities, as well as the Cambridge Supersymmetry Working Group for useful suggestions and discussion related to this paper. We would also like to thank José Zurita for his useful comments and suggestions. This research is supported in part by the Swiss National Science Foundation (SNF) under contract 200020138206 and the Research Executive Agency (REA) of the European Union under the Grant Agreement number PITN-GA-2010-264564 (LHCPhenoNet). 


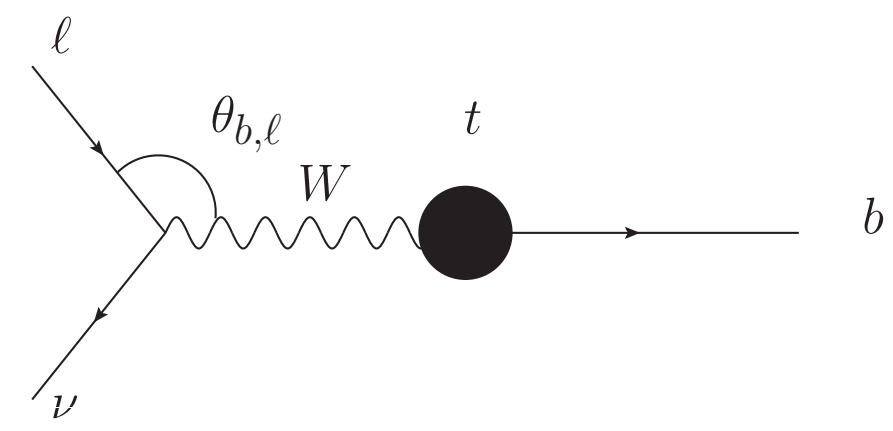

Figure 25. The angle $\theta_{b, \ell}$, between the $b$ quark and lepton in top decay, shown in the centre-ofmass frame of the top for illustration purposes. The angle is calculated in the lab frame throughout this paper.

\section{A Angular variables}

\section{A.1 Definitions}

We define here two 'angular' variables, the first of which is the angle $\theta_{b, \ell}$, defined between the $b$-quark and the lepton, $\ell$, in semi-leptonic top decays as shown in figure 25 . The angle is defined in the lab frame, but is shown in the figure in the centre-of-mass frame of the decaying top quark for illustration purposes. We consider a function of this variable defined by: ${ }^{15}$

$$
f\left(\cos \theta_{b, \ell}\right)=0.25 \times\left(1+\cos \theta_{b, \ell}\right)^{2} .
$$

We will also be considering the distance between the lepton and neutrino for semi-leptonic top decays, given by $\Delta R(\ell, \nu)=\sqrt{\delta \eta_{\ell, \nu}^{2}+\delta \phi_{\ell, \nu}^{2}}$, where $\delta \eta_{\ell, \nu}$ and $\delta \phi_{\ell, \nu}$ are the distances in the pseudo-rapidity, $\left|\eta_{\ell}-\eta_{\nu}\right|$, and transverse plane angle, $\left|\phi_{\ell}-\phi_{\nu}\right|$.

\section{A.2 Angular variables in the $Z^{\prime}$ flavour-changing model}

Figure 26 shows the angular variables at parton-level for the $Z^{\prime}$ model described by the Lagrangian density of eq. (3.1), for a mass of $1.5 \mathrm{TeV}$ at a $14 \mathrm{TeV}$ LHC. In figure 27 we show the reconstructed distributions after Delphes simulation for the minimal set of cuts, and figures 28 and 29 show the corresponding reconstructed distributions for the set of cuts $A$ and $B$, defined in section 3.1.2. Table 3 shows the corresponding $\chi^{2} / N_{\text {d.o.f. }}$ between the left- and right-handed distributions corresponding to the figures. It is evident that these angular variables can provide equivalent magnitudes of discrimination between left- and right-handed top quarks as the energy function variables that have been used throughout the main part of the paper.

\section{B Detector-level smearing}

The probability density functions used for the likelihood method that corrects for likelihood methods are shown in figure 30 and figure 31 with dashed histograms. The solid

\footnotetext{
${ }^{15}$ This form of the function is chosen to resemble the factor $0.25 \times\left(1+\cos \theta_{b, \ell}^{*}\right)^{2}$, where $\theta_{b, \ell}^{*}$ is defined in the $W$ rest frame, which appears in the top quark decay differential cross section.
} 

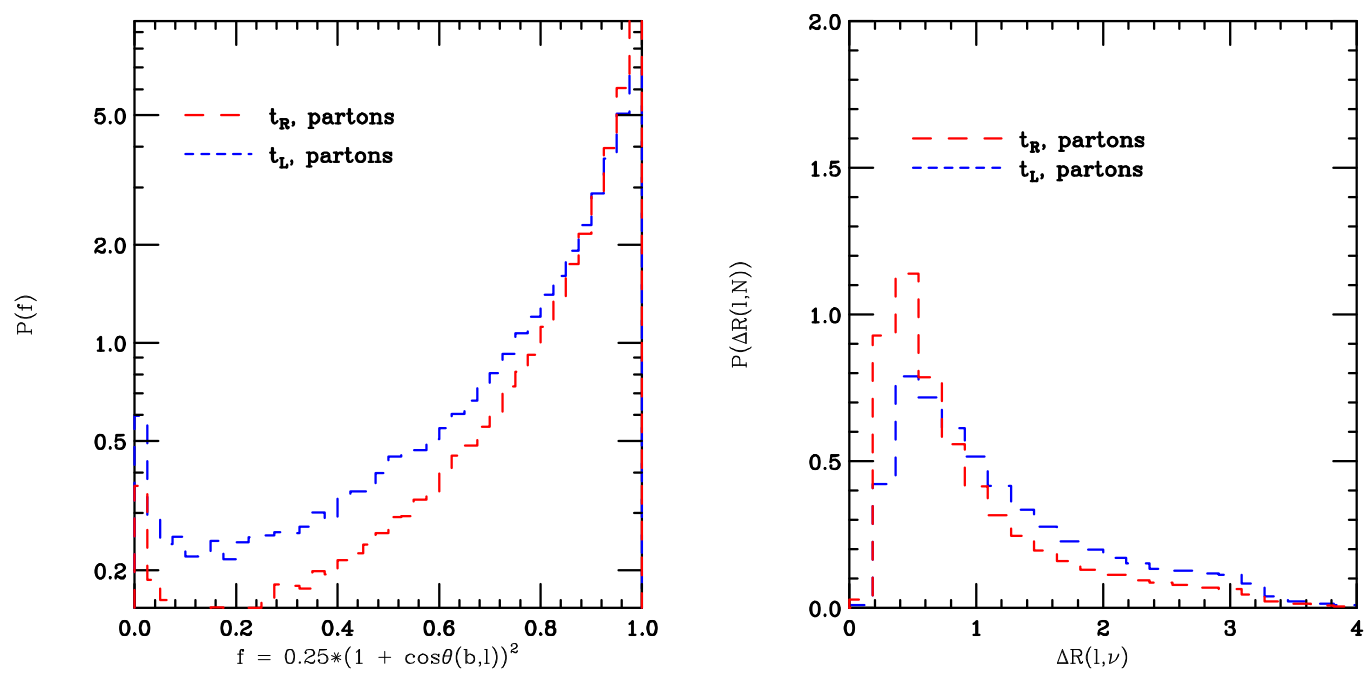

Figure 26. The $f\left(\cos \theta_{b, \ell}\right)$ (left panel) and $\Delta R(\ell, \nu)$ (right panel) variables for left- or right-handed $Z^{\prime}$ bosons decaying to $u \bar{t}$ or $\bar{u}$, obtained from parton-level events.
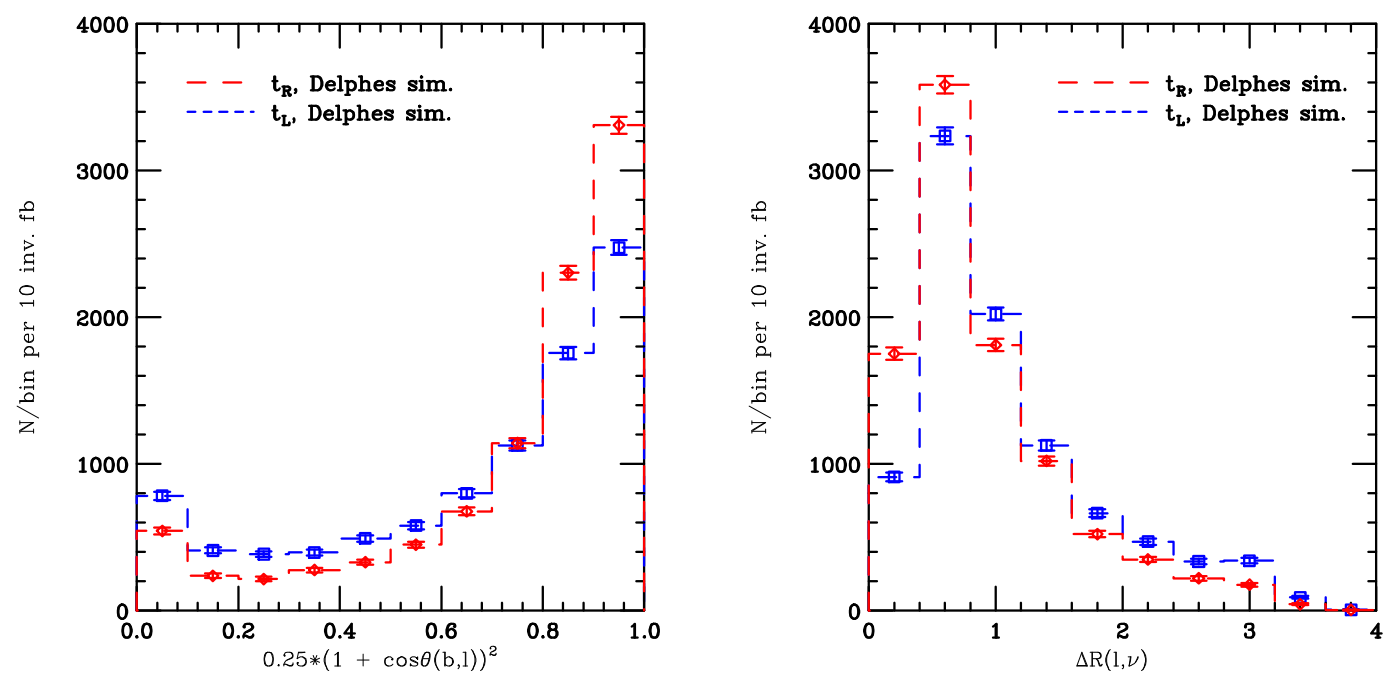

Figure 27. The $f\left(\cos \theta_{b, \ell}\right)$ (left panel) and $\Delta R(\ell, \nu)$ (right panel) variables for a left- or righthanded $Z^{\prime}$ bosons decaying to $u \bar{t}$ or $\bar{u} t$, obtained from the reconstructed events for an LHC run at $14 \mathrm{TeV}$, with $10 \mathrm{fb}^{-1}$.

\begin{tabular}{|c|c|c|}
\hline Variable & $\Delta R(\ell, \nu)$ & $f(\cos \theta(b, \ell))$ \\
\hline Min. & 45.7 & 29.1 \\
\hline A & 35.4 & 19.1 \\
\hline B & 34.0 & 12.6 \\
\hline
\end{tabular}

Table 3. The value of $\chi^{2} / N_{\text {d.o.f. }}$ between the left- and right-handed distributions in the $Z^{\prime}$ model with decays to $u \bar{t}$ or $\bar{u}$, for the three different sets of cuts. It is evident that the distributions are distinguishable even for the higher cuts. 

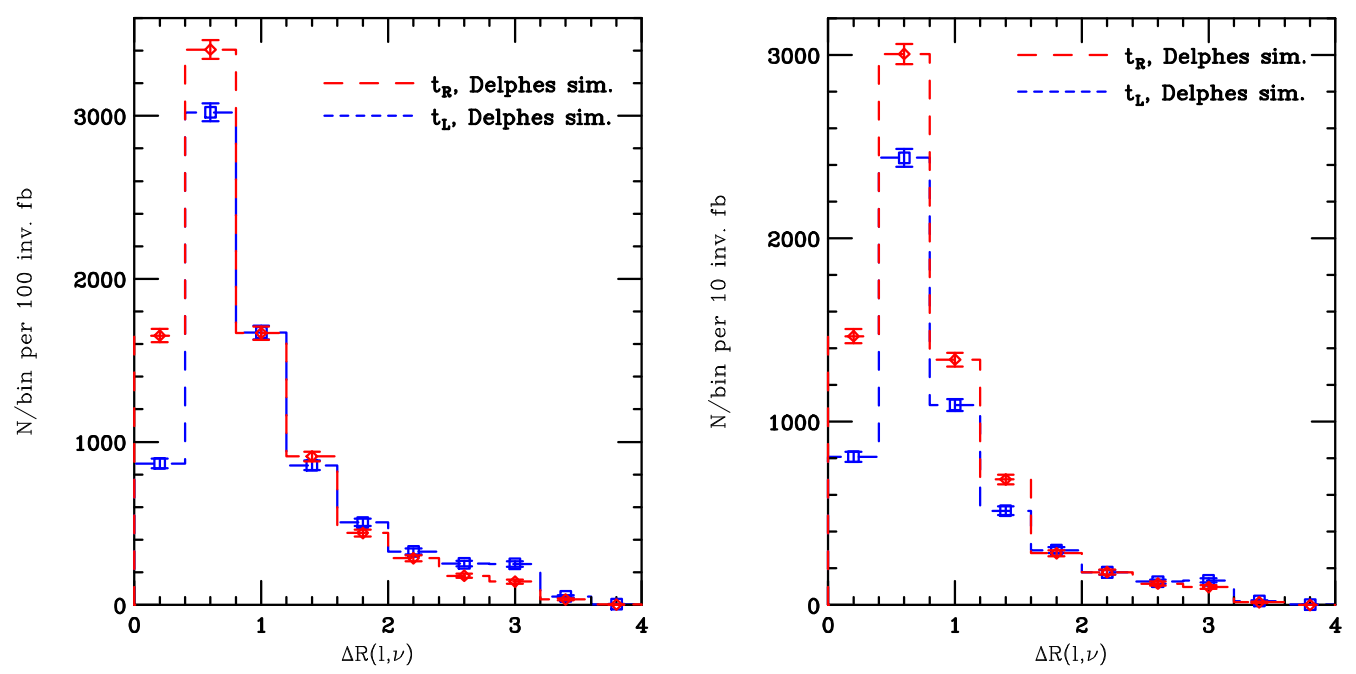

Figure 28. The $\Delta R(\ell, \nu)$ variable for a left- or right-handed $Z^{\prime}$ bosons decaying to $u \bar{t}$ or $\bar{u} t$, obtained from the reconstructed events for an $\mathrm{LHC}$ run at $14 \mathrm{TeV}$, with $10 \mathrm{fb}^{-1}$, with the set of cuts A (left) and B (right), as explained in the text.
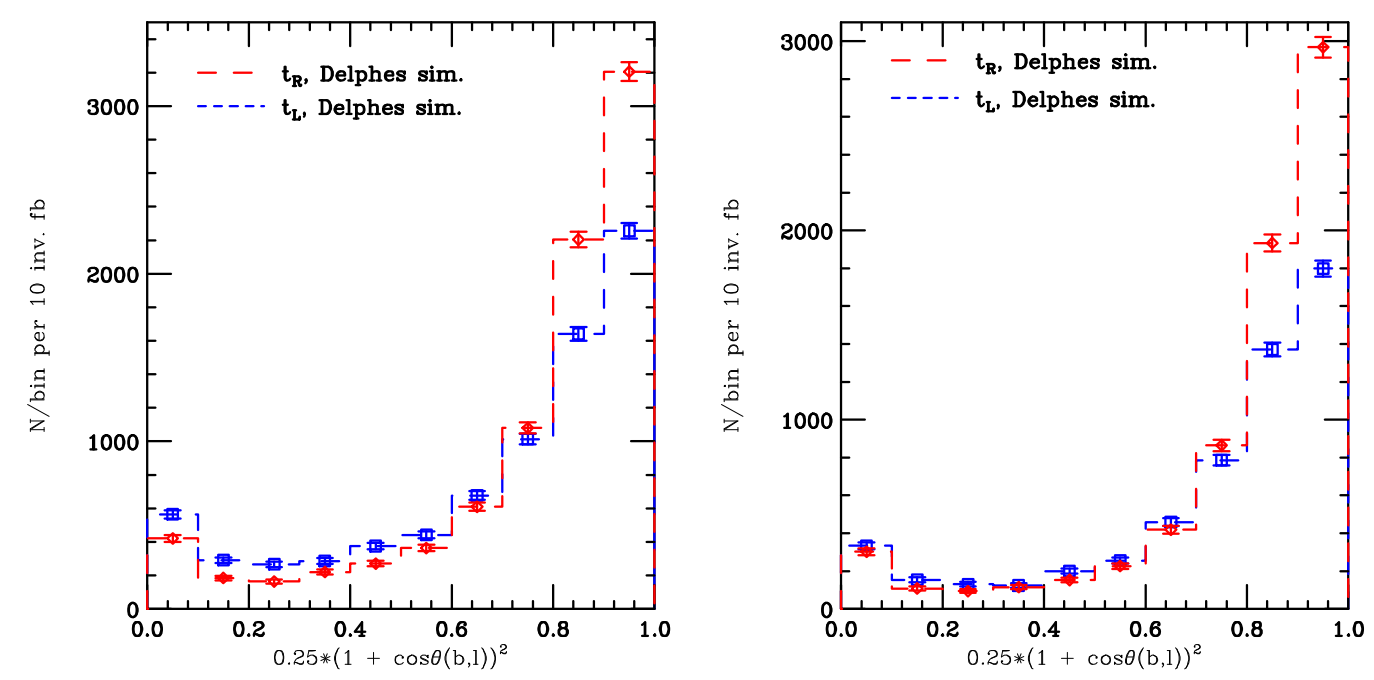

Figure 29. The $f\left(\cos \theta_{b, \ell}\right)$ variable for a left- or right-handed $Z^{\prime}$ bosons decaying to $u \bar{t}$ or $\bar{u} t$, obtained from the reconstructed events for an LHC run at $14 \mathrm{TeV}$, with $10 \mathrm{fb}^{-1}$, with the set of cuts A (left) and B (right), as explained in the text.

histograms show the actual discrepancy between parton-level and detector-level objects as obtained by Delphes. In practice these distributions would be known features of the detector performance.

\section{Alternative leptoquark decay: $S_{X Y} \rightarrow t_{R} \bar{\tau}_{L}, t_{L} \bar{\tau}_{R}$}

In section 3.3 we considered the decay of a leptoquark which we called $S_{X X}$, where $X \in$ $\{L, R\}$, with electromagnetic charge $\pm 5 / 3$ to either $t_{R} \tau_{R}$ or $t_{L} \tau_{L}$. Here we consider the 

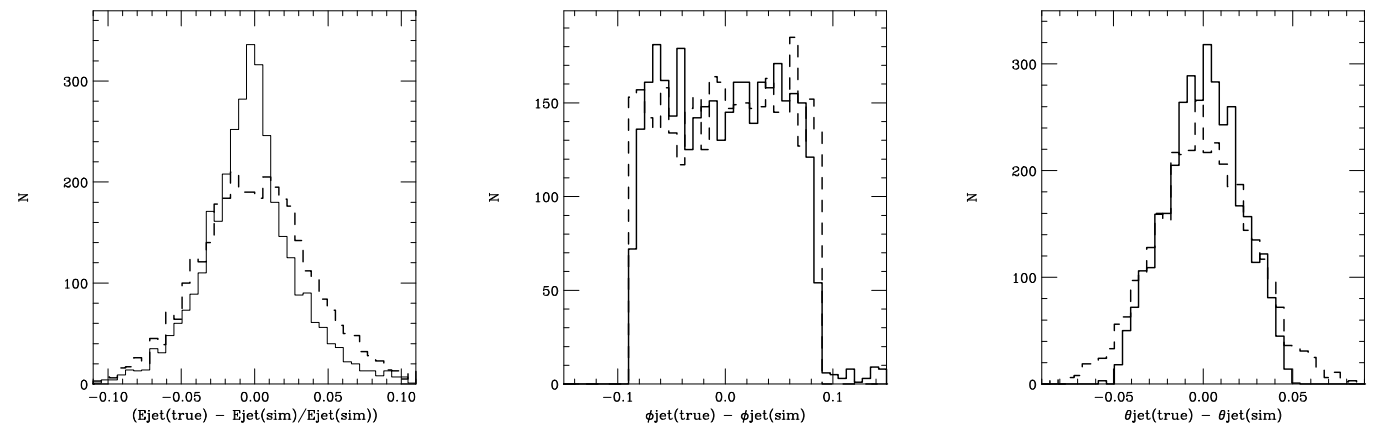

Figure 30. The probability density functions for smearing of jet energy, the angle on the transverse plane $\phi$ of jets, and the azimuthal angle $\theta$ of jets. The solid histograms show the actual Delphes simulation results whereas the dashed one show the functions that were actually used for the likelihood method of section 3.2 .
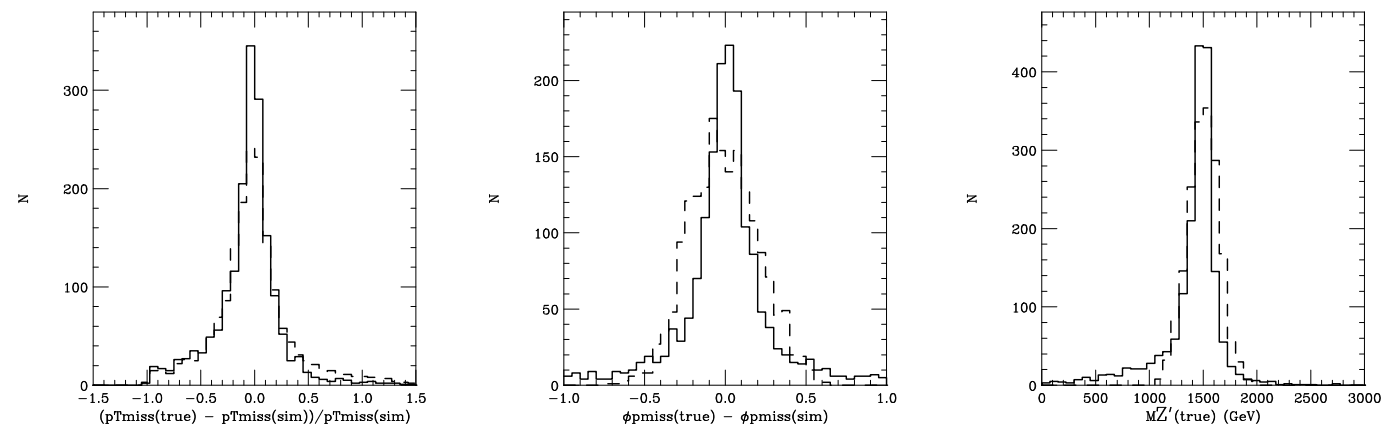

Figure 31. The probability density functions for smearing of missing transverse energy, the angle on the transverse plane, $\phi$, of the missing transverse momentum vector, and the actual $Z^{\prime}$ mass distribution in the model described by the Lagrangian of eq. (3.6) (measured through some other decay mode than the $\tau \tau$ mode). The solid histograms show the actual Delphes simulation results whereas the dashed one show the functions that were actually used for the likelihood method of section 3.2 .

alternative combination of helicities, corresponding to a leptoquark with charge $\pm 1 / 3$ which we call $S_{X Y}$, where $X \neq Y$ and $X, Y \in\{L, R\} .{ }^{16}$ The results for $x_{\text {top }}$ and $x_{\tau}$, corresponding to the fully-hadronic leptoquark analysis constructed in section 3.3 are shown in figure 32 , for the minimal set of cuts. Note that in this case one should understand that the lefthanded $\tau$ should be paired up with the right-handed top and vice versa (i.e. red in one plot with blue in the other).

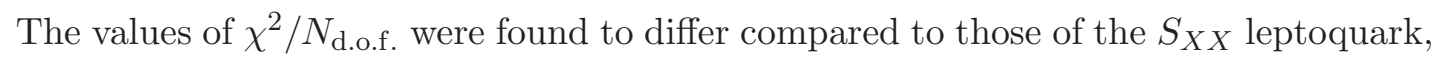
corresponding to $\sim 1.6$ for the $x_{\tau}$ variable and $\sim 8.2$ for the $x_{\text {top }}$ variable. The differences between the $S_{X Y}$ and $S_{X X}$ leptoquarks arise due to the difference in acceptances of the left- and right-handed particles. These are a consequence the interplay of the efficiencies associated with the $b$-tagging, $\tau$-tagging and reconstruction of the top quarks.

\footnotetext{
${ }^{16}$ In the notation of ref. [13], we are actually considering the decays of the upper component of the $S_{1 / 2}$ doublet, $S_{1 / 2}^{(+)}$.
} 

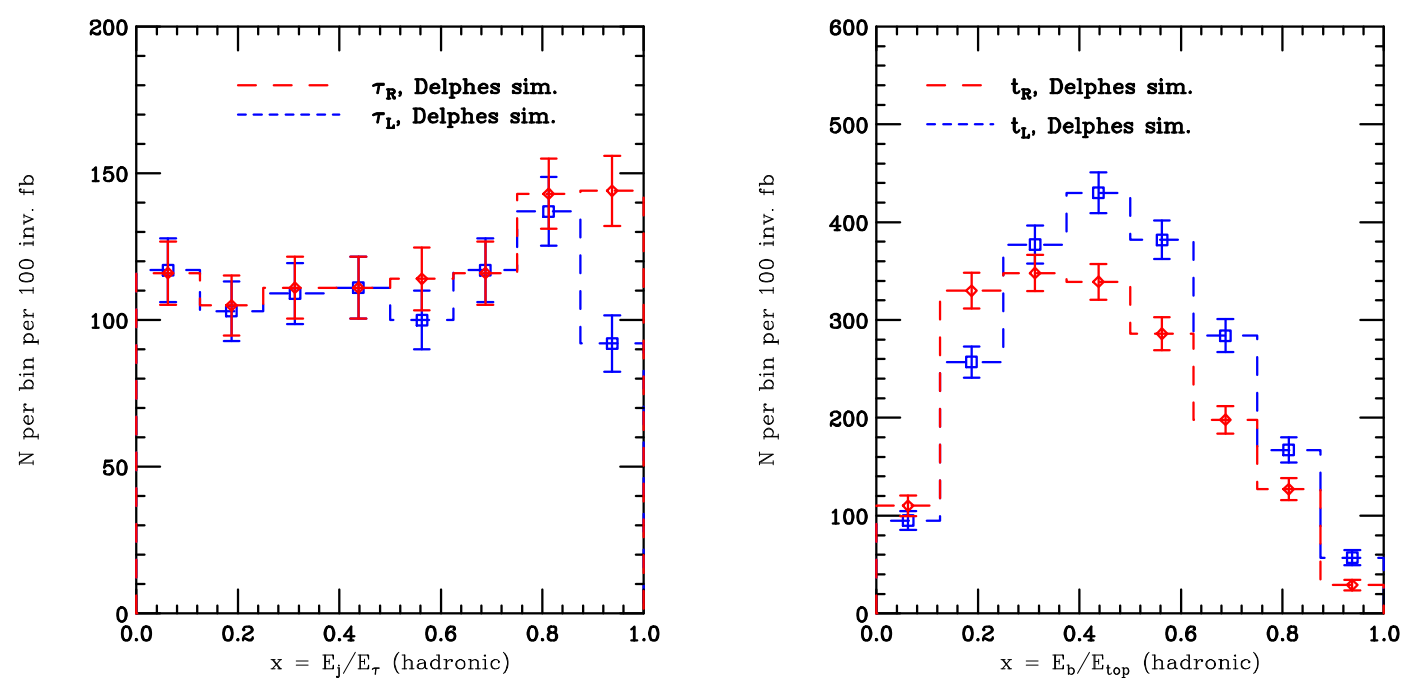

Figure 32. The $x_{\tau}$ (left panel) and $x_{\text {top }}$ (right panel) variables for left- or right-handed tops from the $S_{X Y}$ leptoquark, for an integrated luminosity of $100 \mathrm{fb}^{-1}$ for a $14 \mathrm{TeV}$ LHC.

\section{Matrix element for polarised top decay}

It is useful to outline here the derivation of the polarised top decay differential width. The coupling of the $W$ boson to the fermions is given by:

$$
\mathcal{L}=\frac{g}{2 \sqrt{2}} V_{f f^{\prime}} \bar{f} \gamma_{\mu}\left(1-\gamma_{5}\right) f^{\prime} W^{\mu}+\text { h.c. },
$$

where $g$ is the weak charge and $V_{f f^{\prime}}$ is the CKM matrix element corresponding to the fermions $f$ and $f^{\prime}$. The matrix element, corresponding to the diagram shown in figure 33 is given by:

$$
\mathcal{M}=\frac{g^{2}}{8} V_{t b} V_{f f^{\prime}} \bar{u}_{b}^{r}\left(p_{2}\right) \gamma_{\mu}\left(1-\gamma_{5}\right) W^{\mu} u_{t}^{s} p_{1} \bar{u}_{f^{\prime}}^{r_{1}}\left(q_{1}\right) \gamma_{\nu}\left(1-\gamma_{5}\right) v_{f}^{r_{2}}\left(q_{2}\right) W^{\nu} G\left(q^{2}\right)
$$

where $u$ and $v$ are the positive and negative frequency spinors, $W^{\mu}$ is the $W$ polarisation vector and $G\left(q^{2}\right)$ is the $W$ propagator,

$$
G\left(q^{2}\right)=\frac{1}{\left(q^{2}-m_{W}^{2}\right)+i \Gamma_{W} m_{W}} .
$$

Squaring the matrix element and summing over the $b$ quark and fermion spins, we obtain:

$$
\begin{aligned}
\sum_{r_{1}, r_{2}, r}|\mathcal{M}|= & \Omega \operatorname{Tr}\left[\frac{1}{2}\left(1+2 s \gamma_{5} \not\right)\left(\not p_{1}+m_{\mathrm{top}}\right) \gamma_{k}\left(1-\gamma_{5}\right) \not p_{2} \gamma_{\mu}\left(1-\gamma_{5}\right)\right] \\
& \times \operatorname{Tr}\left[\phi_{1} \gamma_{\nu}\left(1-\gamma_{5}\right) \phi_{2} \gamma_{\lambda}\left(1-\gamma_{5}\right)\right] \\
& \times W^{\mu} W^{\nu} W^{* \lambda} W^{* k}
\end{aligned}
$$




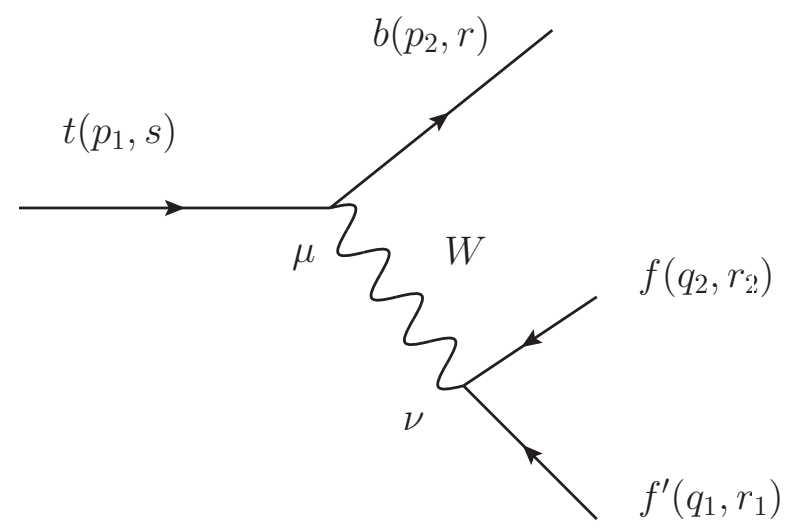

Figure 33. The Feynman diagram for polarised top decay. The parentheses indicate the 4momentum and spin labels respectively, for each particle.

where $S=1 / m_{\text {top }}\left(\left|\vec{p}_{1}\right|, E_{1} \vec{p}_{1} /\left|\vec{p}_{1}\right|\right)$ is the spin 4 -vector for the top-quark and $\Omega$ is defined as:

$$
\Omega \equiv \frac{g^{4}}{64}\left|V_{t b}\right|^{2}\left|V_{f f^{\prime}}\right|^{2} \times \frac{1}{\left(q^{2}-m_{W}^{2}\right)^{2}+\Gamma_{W}^{2} m_{W}^{2}}
$$

The traces can be calculated using the FORM package [28]. The first trace in eq. (D.4), corresponding to a top quark with spin $s= \pm 1 / 2$ and the bottom quarks, is given by:

$$
\begin{aligned}
& 4\left(p_{1}^{\mu} p_{2}^{k}+p_{1}^{k} p_{2}^{\mu}-g^{\mu k} p_{1} \cdot p_{2}-\epsilon^{i j \mu k} p_{1}^{i} p_{2}^{j}\right) \\
+ & 8 m_{\mathrm{top}} s\left(-p_{2}^{\mu} S^{k}-S^{\mu} p_{2}^{k}+g^{\mu k} p_{2} \cdot S-\epsilon^{i j \mu k} p_{2}^{i} S^{j}\right) .
\end{aligned}
$$

It is obvious that the second term in the above result vanishes if we sum over $s$ or set $m_{\text {top }} \rightarrow 0$. The second trace in eq. (D.4), corresponding to the fermions $f$ and $f^{\prime}$ is given by:

$$
8\left(q_{1}^{\nu} q_{2}^{\lambda}+q_{1}^{\lambda} q_{2}^{\nu}-g^{\nu \lambda} q_{1} \cdot q_{2}+8 \epsilon^{i j \mu k} q_{1}^{i} q_{2}^{j}\right) .
$$

Summing over the $W$ polarisations introduces $g^{k \lambda}$ and $g^{\mu \nu}$, and gives, for the polarised top matrix element squared,

$$
|\mathcal{M}|^{2}(s)=128 \Omega\left(p_{2} \cdot q_{1}\right)\left[\left(p_{1}-m_{\mathrm{top}}(2 s) S\right) \cdot q_{2}\right] .
$$

\section{E Finite mass effects on top polarisation}

\section{E.1 Production polarisation}

We adapt the description given in ref. [4] for the case of stop decay to a top and a neutralino, to the decay of a scalar leptoquark to a top and another fermion. The corresponding Lagrangian for the decay of scalar leptoquark $S$ to $t_{L, R}$ and a fermion $f$ can be written as:

$$
\mathcal{L}=g_{L} \bar{t}_{L} S f+g_{R} \bar{t}_{R} S f+\text { h.c. },
$$


where $g_{L}$ and $g_{R}$ are the left- and right-handed couplings respectively. The axis of spin quantisation is taken to lie along the top direction of motion in the parent leptoquark rest frame. The production amplitudes for positive and negative helicity top quarks depend on two functions $F_{ \pm}$:

$$
F_{ \pm}=\frac{\left(E_{t}+m_{\mathrm{top}} \pm\left|p_{t}\right|\right)\left(E_{f}+m_{f} \pm\left|p_{f}\right|\right)}{\sqrt{4\left(E_{t}+m_{\mathrm{top}}\right)\left(E_{f}+m_{f}\right)}}
$$

where all quantities are given in the leptoquark rest frame. The functions result from explicit evaluation of the matrix element for the leptoquark decay using the spinor wavefunctions. These are given by:

$$
u(\vec{p}, s)=\left(\begin{array}{c}
\sqrt{p \cdot \sigma} \chi_{s} \\
\sqrt{p \cdot \bar{\sigma}} \chi_{s}
\end{array}\right), v(\vec{p}, s)=\left(\begin{array}{c}
2 s \sqrt{p \cdot \sigma} \chi_{-s} \\
-2 s \sqrt{p \cdot \bar{\sigma}} \chi_{-s}
\end{array}\right)
$$

where the spinors $\chi_{s}$ are eigenstates of the operator $1 / 2 \vec{\sigma} \cdot \hat{\vec{s}}$ with eigenvalue $s$, where $s= \pm 1 / 2$ corresponding to spin-up and spin-down respectively. The spinors are given, for $\hat{\vec{s}}$ along the $z$-direction, by:

$$
\chi_{1 / 2}(\hat{\vec{z}})=\left(\begin{array}{l}
1 \\
0
\end{array}\right), \quad \chi_{-1 / 2}(\hat{\vec{z}})=\left(\begin{array}{l}
0 \\
1
\end{array}\right) .
$$

For the decay of a scalar to left-handed tops, for example, the matrix element is proportional to $\bar{v}(\vec{p}, s) P_{L} u(\vec{k}, r)$, where $\vec{p}$ and $s$ are the top momentum and spin respectively, and $\vec{k}$ and $r$ are the fermion momentum and spin respectively. The $P_{L}$ operator projects the upper component of $u(\vec{k}, r)$ and hence the matrix element for the left-handed coupling is given by:

$$
\mathcal{M}_{s r}^{L} \propto\left(\begin{array}{ll}
-2 s \sqrt{p \cdot \bar{\sigma}} \chi_{-s}^{\dagger} 2 s \sqrt{p \cdot \sigma} \chi_{-s}^{\dagger}
\end{array}\right)\left(\begin{array}{c}
\sqrt{k \cdot \sigma} \chi_{r} \\
0
\end{array}\right)
$$

Using the relations [29]:

$$
\begin{aligned}
\sqrt{p \cdot \sigma} & =\frac{\left(E_{p}+m\right) \mathbb{I}-\vec{\sigma} \cdot \vec{p}}{\sqrt{2\left(E_{p}+m\right)}}, \\
\sqrt{p \cdot \bar{\sigma}} & =\frac{\left(E_{p}+m\right) \mathbb{I}+\vec{\sigma} \cdot \vec{p}}{\sqrt{2\left(E_{p}+m\right)}}
\end{aligned}
$$

we obtain the following:

$$
\mathcal{M}_{s r}^{L} \propto-2 s \chi_{-s}^{\dagger} \frac{\left[\left(E_{t}+m_{\mathrm{top}}\right) \mathbb{I}+\vec{\sigma} \cdot \vec{p}\right]\left[\left(E_{f}+m_{f}\right) \mathbb{I}-\vec{\sigma} \cdot \vec{k}\right]}{\sqrt{4\left(E_{t}+m_{\mathrm{top}}\right)\left(E_{f}+m_{f}\right)}} \chi_{r}
$$

or, writing out the matrices explicitly:

$$
\mathcal{M}_{s r}^{L} \propto-\frac{2 s}{D} \chi_{-s}^{\dagger}\left(\begin{array}{cc}
E_{t}+m_{\mathrm{top}}+p_{z} & p_{x}-i p_{y} \\
p_{x}+i p_{y} & E_{t}+m_{\mathrm{top}}-p_{z}
\end{array}\right)\left(\begin{array}{cc}
E_{f}+m_{f}-k_{z} & -k_{x}+i k_{y} \\
k_{x}+i k_{y} & E_{f}+m_{f}+k_{z}
\end{array}\right) \chi_{r}
$$

where we have defined the denominator as $D=\sqrt{4\left(E_{t}+m_{\mathrm{top}}\right)\left(E_{f}+m_{f}\right)}$. In the rest frame of the scalar leptoquark, we can take the $z$-axis to lie along the top direction of 
motion, and hence we have $p_{x}=p_{y}=k_{z}=k_{y}=0$ and $p_{z}=-k_{z}$ and the matrix element becomes:

$$
\mathcal{M}_{s r}^{L} \propto-\frac{2 s}{D} \chi_{-s}^{\dagger}\left(\begin{array}{cc}
E_{t}+m_{\mathrm{top}}+\left|p_{z}\right| & 0 \\
0 & E_{t}+m_{\mathrm{top}}-\left|p_{z}\right|
\end{array}\right)\left(\begin{array}{cc}
E_{f}+m_{f}+\left|k_{z}\right| & 0 \\
0 & E_{f}+m_{f}-\left|k_{z}\right|
\end{array}\right) \chi_{r},
$$

Performing the matrix multiplication, one immediately notices that the functions $F_{ \pm}$appear as elements of the resulting matrix:

$$
\mathcal{M}_{s r}^{L} \propto-2 s \chi_{-s}^{\dagger}\left(\begin{array}{cc}
F_{+} & 0 \\
0 & F_{-}
\end{array}\right) \chi_{r}
$$

If we choose $s=+1 / 2$ then:

$$
\mathcal{M}_{+r}^{L} \propto-\left(0, F_{-}\right) \chi_{r}
$$

or, if we choose $s=-1 / 2$ then:

$$
\mathcal{M}_{-r}^{L} \propto-\left(F_{+}, 0\right) \chi_{r}
$$

Upon squaring and summing over the fermion spin, $r$, we obtain:

$$
\sum_{r}\left|\mathcal{M}_{+r}^{L}\right|^{2}=\left|\mathcal{M}_{+-}^{L}\right|^{2} \propto\left|g_{L} F_{+}\right|^{2}
$$

and

$$
\sum_{r}\left|\mathcal{M}_{-r}^{L}\right|^{2}=\left|\mathcal{M}_{-+}^{L}\right|^{2} \propto\left|g_{L} F_{-}\right|^{2}
$$

Since only one component of $\left|\mathcal{M}_{s r}^{L}\right|$ contributes, we may also write:

$$
\mathcal{M}_{ \pm}^{L} \equiv \mathcal{M}_{ \pm \mp}^{L}
$$

and hence:

$$
\mathcal{M}_{ \pm}^{L} \propto g_{L} F_{\mp} .
$$

The extension to $\mathcal{M}_{ \pm}^{R}$ is trivial, for which the resulting matrix elements are thus given by:

$$
\mathcal{M}_{ \pm}^{R} \propto g_{R} F_{ \pm}
$$

For finite $m_{\text {top }}$ this gives a non-vanishing amplitude for top quarks of both helicities even in the limit of a purely chiral vertex. It can be shown that the polarisation along the production axis at a parent rest frame is given by:

$$
\begin{aligned}
\left\langle P_{P}\right\rangle & \equiv \frac{\left|\mathcal{M}_{+}^{L}+\mathcal{M}_{+}^{R}\right|^{2}-\left|\mathcal{M}_{-}^{L}+\mathcal{M}_{-}^{R}\right|^{2}}{\left|\mathcal{M}_{+}^{L}+\mathcal{M}_{+}^{R}\right|^{2}+\left|\mathcal{M}_{-}^{L}+\mathcal{M}_{-}^{R}\right|^{2}} \\
& =\frac{\left(\left|g_{R}\right|^{2}-\left|g_{L}\right|^{2}\right) M_{\mathrm{LQ}}\left|p_{t}\right|}{\left(\left|g_{R}\right|^{2}+\left|g_{L}\right|^{2}\right)\left(M_{\mathrm{LQ}} E_{t}-m_{\mathrm{top}}^{2}\right)+2 g_{R} g_{L} m_{f} m_{\mathrm{top}}},
\end{aligned}
$$



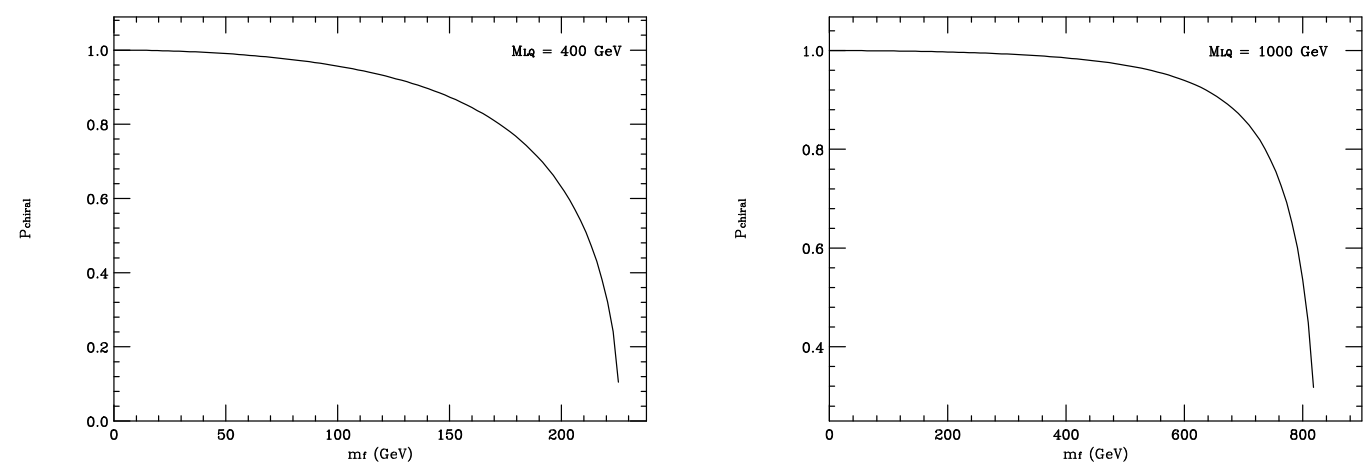

Figure 34. The effect of the sister fermion mass $m_{f}$ on the purely chiral top quark polarisation, $\left\langle P_{P}\right\rangle_{\text {chiral }}$, in the decay of a scalar leptoquark of mass $M_{L Q}=400 \mathrm{GeV}$ (left panel), or $M_{L Q}=$ $1000 \mathrm{GeV}$ (right panel), to $t f$.

where

$$
\begin{aligned}
& \left|p_{t}\right|=\frac{\sqrt{\left(M_{\mathrm{LQ}}^{2}+m_{\mathrm{top}}^{2}-m_{f}^{2}\right)^{2}-4 M_{\mathrm{LQ}}^{2} m_{\mathrm{top}}^{2}}}{2 M_{\mathrm{LQ}}}, \\
& E_{t}=\frac{M_{\mathrm{LQ}}^{2}-m_{f}^{2}+m_{\mathrm{top}}^{2}}{2 M_{\mathrm{LQ}}}
\end{aligned}
$$

and $M_{\mathrm{LQ}}$ is the parent leptoquark mass. It is interesting to consider the effect of the mass of the sister fermion produced in the decay in the purely chiral case. Setting either $g_{R}=0$ or $g_{L}=0$ defines:

$$
\left\langle P_{P}\right\rangle_{\text {chiral }} \equiv\left\langle P_{P}\right\rangle_{\left(g_{R} \text { or } g_{L}=0\right)}= \pm \frac{M_{\mathrm{LQ}}\left|p_{t}\right|}{M_{\mathrm{LQ}} E_{t}-m_{\mathrm{top}}^{2}}
$$

If we had $m_{f}=m_{\text {top }}=0$, then we would get $\left\langle P_{P}\right\rangle_{\text {chiral }}= \pm 1$. For a non-zero top quark mass, it turns out that for a wide range of values of $m_{f}$, the deviation from the value of $\left\langle P_{P}\right\rangle_{\text {chiral }}= \pm 1$ is small. Figure 34 shows the effect of the fermion mass for the case $M_{L Q}=400 \mathrm{GeV}$ and $M_{L Q}=1000 \mathrm{GeV}$. It is clear that when $m_{f} \ll m_{\text {top }}$, then the effect is small, and $\left\langle P_{P}\right\rangle_{\text {chiral }}$ is close to the $m_{f}=0$ value. The effect is $\mathcal{O}$ (few \%) even for $m_{f} \sim 100 \mathrm{GeV}$ in the $M_{L Q}=400 \mathrm{GeV}$ case. For $M_{L Q}=1000 \mathrm{GeV}$, the effect of the sister particle mass is of $\mathcal{O}\left(\right.$ few $\%$ ) even for masses as high as $\sim 600 \mathrm{GeV} .^{17}$

One may also consider effects of relativistic rotation via the Wigner angle between the production and detection axes of the top quark [4]. However, if the velocity of parent particle is small or the top is highly boosted in the parent rest frame, the effect of the Wigner rotation is negligible. Therefore we can ignore it for our $Z^{\prime}$ examples, since we have assumed $Z^{\prime}$ being much heavier than the daughter particles. Even for the leptoquark example, the effect has been checked and found not to be significant, since most of the events have $\beta_{p} \ll 1$ and $\beta_{t} \sim 1$, as we will see in the next subsection.

\footnotetext{
${ }^{17}$ Note that it may be interesting to investigate whether the helicity distributions of the top can provide independent information on the mass of the accompanying sister particle, especially if this is weaklyinteracting (and hence invisible).
} 


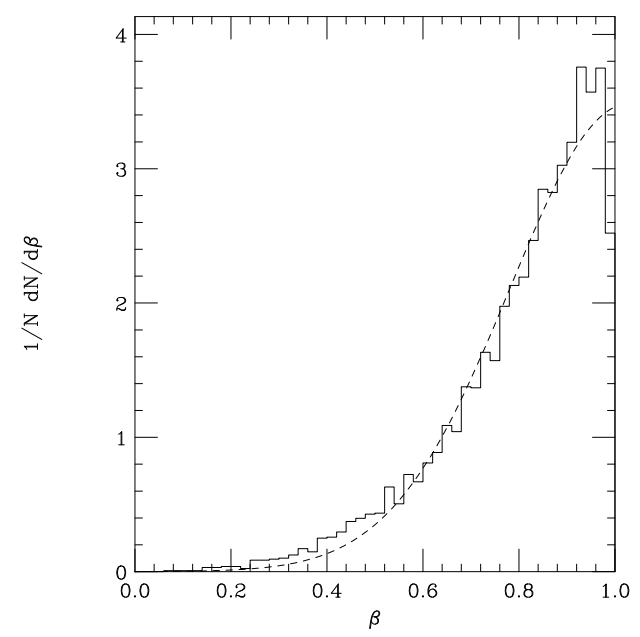

Figure 35. The fit to the top quark $\beta_{t}$ distribution for $400 \mathrm{GeV}$ leptoquarks is shown in solid black dashes. The HeRWIG++ histogram extracted from parton-level Monte Carlo events is shown in solid black.

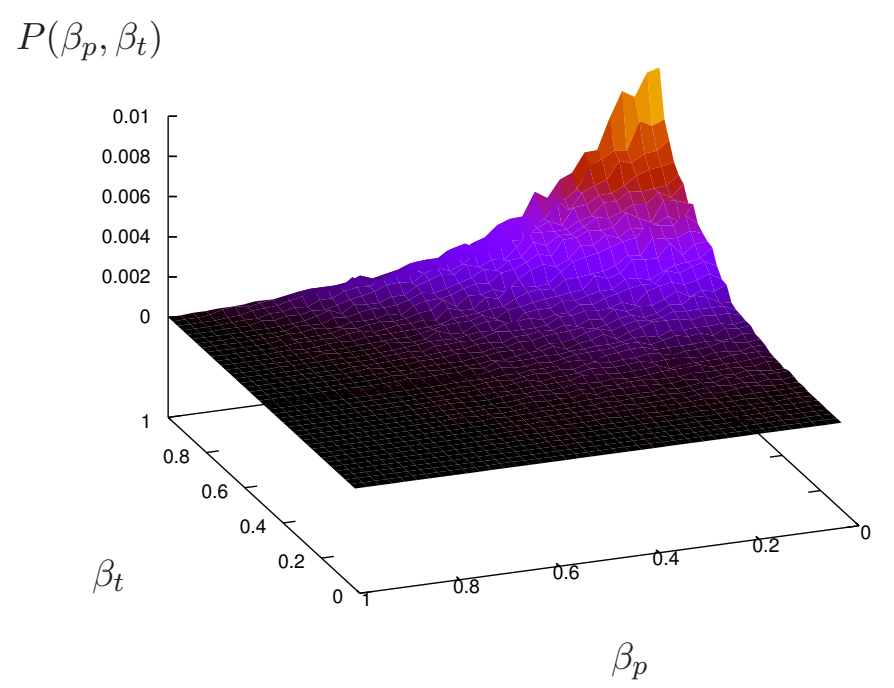

Figure 36. The $\beta_{p}-\beta_{t}$ distribution for $400 \mathrm{GeV}$ leptoquarks, extracted from the HERWIG++ event generator, is shown.

\section{E.2 $\beta$ distributions from Monte Carlo}

We also show the form of the two-dimensional $\beta_{p}-\beta_{t}$ distribution for $400 \mathrm{GeV}$ leptoquarks, extracted from the HERWIG++ event generator in figure 36. For the $\beta_{t}$ distribution (i.e. integrated over $\beta_{p}$ ) in the decay of a $400 \mathrm{GeV}$ leptoquark a fit can be made, shown in figure 35 . The fit has the form of a Gaussian:

$$
P\left(\beta_{t}\right)=a \exp \left(-\left(\beta_{t}-b\right)^{2} / c\right),
$$

where the parameters $a, b$ and $c$ were given by the fit to be $a \simeq 3.48, b \simeq 1.03, c \simeq 0.13$. The distribution integrates to $\sim 1$ in $\beta_{t} \in(0,1)$. 
Open Access. This article is distributed under the terms of the Creative Commons Attribution License which permits any use, distribution and reproduction in any medium, provided the original author(s) and source are credited.

\section{References}

[1] B. Gripaios, Composite leptoquarks at the LHC, JHEP 02 (2010) 045 [arXiv:0910.1789] [INSPIRE].

[2] M. Jezabek and J.H. Kuhn, Lepton spectra from heavy quark decay, Nucl. Phys. B 320 (1989) 20 [INSPIRE].

[3] A. Czarnecki, M. Jezabek and J.H. Kuhn, Lepton spectra from decays of polarized top quarks, Nucl. Phys. B 351 (1991) 70 [InSPIRE].

[4] J. Shelton, Polarized tops from new physics: signals and observables, Phys. Rev. D 79 (2009) 014032 [arXiv:0811.0569] [INSPIRE].

[5] B. Bullock, K. Hagiwara and A.D. Martin, Tau polarization as a signal of charged Higgs bosons, Phys. Rev. Lett. 67 (1991) 3055 [INSPIRE].

[6] B.K. Bullock, K. Hagiwara and A.D. Martin, Tau polarization and its correlations as a probe of new physics, Nucl. Phys. B 395 (1993) 499 [INSPIRE].

[7] J.D. Anderson, M.H. Austern and R.N. Cahn, Measurement of $Z^{\prime}$ couplings at future hadron colliders through decays to $\tau$ leptons, Phys. Rev. D 46 (1992) 290 [InSPIRE].

[8] S. Gieseke et al., HERWIG++ 2.5 release note, arXiv:1102.1672 [INSPIRE].

[9] D0 collaboration, V.M. Abazov et al., Forward-backward asymmetry in top quark-antiquark production, Phys. Rev. D 84 (2011) 112005 [arXiv:1107.4995] [INSPIRE].

[10] CDF collaboration, T. Aaltonen et al., Evidence for a mass dependent forward-backward asymmetry in top quark pair production, Phys. Rev. D 83 (2011) 112003 [arXiv:1101.0034] [INSPIRE].

[11] M. Duraisamy, A. Rashed and A. Datta, The top forward backward asymmetry with general $Z^{\prime}$ couplings, Phys. Rev. D 84 (2011) 054018 [arXiv:1106.5982] [InSPIRE].

[12] M.R. Buckley, D. Hooper, J. Kopp and E. Neil, Light $Z^{\prime}$ bosons at the Tevatron, Phys. Rev. D 83 (2011) 115013 [arXiv:1103.6035] [INSPIRE].

[13] B. Gripaios, A. Papaefstathiou, K. Sakurai and B. Webber, Searching for third-generation composite leptoquarks at the LHC, JHEP 01 (2011) 156 [arXiv: 1010.3962] [INSPIRE].

[14] A. Brandenburg, Z. Si and P. Uwer, QCD corrected spin analyzing power of jets in decays of polarized top quarks, Phys. Lett. B 539 (2002) 235 [hep-ph/0205023] [INSPIRE].

[15] M. Jezabek, Top quark physics, Nucl. Phys. Proc. Suppl. 37B (1994) 197 [hep-ph/9406411] [INSPIRE].

[16] W. Bernreuther, Top quark physics at the LHC, J. Phys. G 35 (2008) 083001 [arXiv: 0805.1333] [INSPIRE].

[17] R.M. Godbole, K. Rao, S.D. Rindani and R.K. Singh, On measurement of top polarization as a probe of $t \bar{t}$ production mechanisms at the LHC, JHEP 11 (2010) 144 [arXiv:1010.1458] [INSPIRE]. 
[18] R.M. Godbole, L. Hartgring, I. Niessen and C.D. White, Top polarisation studies in $H^{-} t$ and Wt production, JHEP 01 (2012) 011 [arXiv:1111.0759] [INSPIRE].

[19] ATLAS collaboration, G. Aad et al., Search for new phenomena in ttbar events with large missing transverse momentum in proton-proton collisions at $\sqrt{s}=7 \mathrm{TeV}$ with the ATLAS detector, Phys. Rev. Lett. 108 (2012) 041805 [arXiv:1109.4725] [InSPIRE].

[20] CMS collaboration, S. Chatrchyan et al., Search for a vector-like quark with charge $2 / 3$ in $t+Z$ Events from pp collisions at $\sqrt{s}=7 \mathrm{TeV}$, Phys. Rev. Lett. 107 (2011) 271802 [arXiv: 1109.4985] [INSPIRE].

[21] S. Ovyn, X. Rouby and V. Lemaitre, DELPHES, a framework for fast simulation of a generic collider experiment, arXiv:0903.2225 [INSPIRE].

[22] P. Bellan, Measurements of inclusive b-quark production at $7 \mathrm{TeV}$ with the CMS experiment, arXiv:1109.2003 [INSPIRE].

[23] G.P. Salam, Towards jetography, Eur. Phys. J. C 67 (2010) 637 [arXiv:0906.1833] [INSPIRE].

[24] W.H. Press, S.A. Teukolsky, W. T. Vetterling and B. P. Flannery, Numerical recipes: the art of scientific computing, Cambridge University Press, Cambridge U.K. (2007).

[25] A. Elagin, P. Murat, A. Pranko and A. Safonov, A new mass reconstruction technique for resonances decaying to di- $\tau$, Nucl. Instrum. Meth. A 654 (2011) 481 [arXiv:1012.4686] [INSPIRE].

[26] B. Gripaios, K. Sakurai and B. Webber, Polynomials, Riemann surfaces and reconstructing missing-energy events, JHEP 09 (2011) 140 [arXiv:1103.3438] [INSPIRE].

[27] T. Plehn, M. Spannowsky and M. Takeuchi, How to improve top tagging, Phys. Rev. D 85 (2012) 034029 [arXiv:1111.5034] [INSPIRE].

[28] J. Vermaseren, New features of FORM, math-ph/0010025 [INSPIRE].

[29] H.K. Dreiner, H.E. Haber and S.P. Martin, Two-component spinor techniques and Feynman rules for quantum field theory and supersymmetry, Phys. Rept. 494 (2010) 1

[arXiv: 0812.1594] [INSPIRE]. 